\title{
UNIFIED PRIMAL FORMULATION-BASED A PRIORI AND A POSTERIORI ERROR ANALYSIS OF MIXED FINITE ELEMENT METHODS
}

\author{
MARTIN VOHRALÍK
}

\begin{abstract}
We derive in this paper a unified framework for a priori and a posteriori error analysis of mixed finite element discretizations of second-order elliptic problems. It is based on the classical primal weak formulation, the postprocessing of the potential proposed in [T. Arbogast and Z. Chen, On the implementation of mixed methods as nonconforming methods for secondorder elliptic problems, Math. Comp. 64 (1995), 943-972], and the discrete Friedrichs inequality. Our analysis in particular avoids any explicit use of the uniform discrete inf-sup condition and in a straightforward manner and under minimal necessary assumptions, known convergence and superconvergence results are recovered. The same framework then turns out to lead to optimal a posteriori energy error bounds. In particular, estimators for all families and orders of mixed finite element methods on grids consisting of simplices or rectangular parallelepipeds are derived. They give a guaranteed and fully computable upper bound on the energy error, represent error local lower bounds, and are robust under some conditions on the diffusion-dispersion tensor. They are thus suitable for both overall error control and adaptive mesh refinement. Moreover, the developed abstract framework and a posteriori error estimates are quite general and apply to any locally conservative method. We finally prove that in parallel and simultaneously in converse to Galerkin finite element methods, under some circumstances, the weak solution is the orthogonal projection of the postprocessed mixed finite element approximation onto the $H_{0}^{1}(\Omega)$ space and also establish several links between mixed finite element approximations and some generalized weak solutions.
\end{abstract}

\section{INTRODUCTION}

We consider in this paper the model problem

$$
\begin{aligned}
-\nabla \cdot(\mathbf{S} \nabla p)=f & \text { in } \Omega, \\
p=0 & \text { on } \partial \Omega,
\end{aligned}
$$

where $\Omega \subset \mathbb{R}^{d}, d=2,3$, is a polygonal (polyhedral) domain (open, bounded, and connected set), $\mathbf{S}$ is a symmetric, bounded, and uniformly positive definite tensor,

Received by the editor July 7, 2008 and, in revised form, August 6, 2009.

2010 Mathematics Subject Classification. Primary 65N15, 65N30, 76 S05.

Key words and phrases. Mixed finite element methods, a priori error estimates, inf-sup condition, postprocessing, discrete Friedrichs inequality, locally conservative methods, a posteriori error estimates, guaranteed upper bound, orthogonal projection, generalized weak solution.

This work was supported by the GNR MoMaS project "Numerical Simulations and Mathematical Modeling of Underground Nuclear Waste Disposal", PACEN/CNRS, ANDRA, BRGM, CEA, EdF, IRSN, France. 
and $f \in L^{2}(\Omega)$. The classical primal weak formulation consists in finding $p \in H_{0}^{1}(\Omega)$ such that

$$
(\mathbf{S} \nabla p, \nabla \varphi)=(f, \varphi) \quad \forall \varphi \in H_{0}^{1}(\Omega)
$$

(see Section 2.1 below for the details on the notation). The problem (1.1a)-(1.1b) can be equivalently written as the first-order system

$$
\begin{aligned}
\mathbf{u} & =-\mathbf{S} \nabla p & & \text { in } \Omega, \\
\nabla \cdot \mathbf{u} & =f & & \text { in } \Omega, \\
p & =0 & & \text { on } \partial \Omega,
\end{aligned}
$$

which leads to the weak mixed formulation, consisting in finding $\mathbf{u} \in \mathbf{H}(\operatorname{div}, \Omega)$ and $p \in L^{2}(\Omega)$ such that

$$
\begin{aligned}
\left(\mathbf{S}^{-1} \mathbf{u}, \mathbf{v}\right)-(p, \nabla \cdot \mathbf{v}) & =0 & & \forall \mathbf{v} \in \mathbf{H}(\operatorname{div}, \Omega), \\
(\nabla \cdot \mathbf{u}, \phi) & =(f, \phi) & & \forall \phi \in L^{2}(\Omega) .
\end{aligned}
$$

Note that this formulation is equivalent to (1.2) in the sense that $p=p$ and $\mathbf{u}=$ $-\mathbf{S} \nabla p$, which is straightforward to show; cf. Quarteroni and Valli [52, Section 7.1].

We are interested in mixed finite element approximations to (1.4a) 1.4b), which consist in finding $\mathbf{u}_{h} \in \mathbf{V}_{h}$ and $p_{h} \in \Phi_{h}$ such that

$$
\begin{aligned}
\left(\mathbf{S}^{-1} \mathbf{u}_{h}, \mathbf{v}_{h}\right)-\left(p_{h}, \nabla \cdot \mathbf{v}_{h}\right) & =0 & & \forall \mathbf{v}_{h} \in \mathbf{V}_{h}, \\
\left(\nabla \cdot \mathbf{u}_{h}, \phi_{h}\right) & =\left(f, \phi_{h}\right) & & \forall \phi_{h} \in \Phi_{h} .
\end{aligned}
$$

Here $\Phi_{h} \subset L^{2}(\Omega)$ and $\mathbf{V}_{h} \subset \mathbf{H}(\operatorname{div}, \Omega)$ are some of the usual finite-dimensional spaces defined on a mesh $\mathcal{T}_{h}$ of simplices or rectangular parallelepipeds; see Section 4.1 below and Brezzi and Fortin 20] or Roberts and Thomas [56. The main purposes of this paper are the following: i) present a unified framework for both a priori and a posteriori error analysis of mixed finite element methods; ii) base this framework entirely on the primal weak formulation (1.2) (and its above-cited direct equivalence with (1.4a) - 1.4b) on the continuous level and on postprocessing and the discrete Friedrichs inequality on the discrete level; in particular, the explicit use of the uniform-in- $h$ discrete inf-sup condition is avoided; iii) arrive at optimal a priori estimates (under minimal necessary assumptions); iv) present new (and optimal) a posteriori error estimates; v) obtain these results with as simple as possible proofs; vi) present some new (to the best of the author's knowledge) properties of the mixed finite element methods; vii) give a general framework for a posteriori error estimation in locally conservative methods.

A priori error estimates for mixed finite element methods are usually obtained by means of the saddle-point theory of Brezzi 17] and Babuška 12. Traditionally, the natural norms of the spaces $\mathbf{H}(\operatorname{div}, \Omega)$ and $L^{2}(\Omega)$ are used, but mesh-dependent norms can be employed instead; cf. Babuška et al. [10. Postprocessing of $p_{h}$ into a new approximation $\tilde{p}_{h}$ is then usually used for the double purpose of giving an improved approximation to $p$ and facilitating the implementation of mixed methods; cf. Arnold and Brezzi [9], Bramble and Xu [16], Stenberg [57, Chen [25], and Arbogast and Chen [8]. In combination with mesh-dependent norms, it has also previously been used in order to obtain error estimates in, e.g., Lovadina and Stenberg [44; see also the references therein. Some complementary results are presented by Marini and Pietra [45] and in [25] and [8]. Links between the mixed finite element and nonconforming finite element methods are then, in particular, given 
in 9, 25, 8, Marini [46, Chen [26], or [37, 65]. Recently, Cockburn and Gopalakrishnan [28, 29] showed that analysis of mixed methods can be entirely based on the hybridization (cf. Section 4.3 below) and lifting operators and demonstrated interesting relations between the different mixed methods. Let us also mention that very tight links between mixed finite element and finite volume methods exists; see Younès et al. [68] and 60] and the references therein.

A posteriori error estimates for mixed finite element methods were started in the works of Alonso [7, Braess and Verfürth [15, Carstensen [23, Hoppe and Wohlmuth [39, Achchab et al. 2], Wohlmuth and Hoppe [67, Carstensen and Bartels [24, Kirby [42, El Alaoui and Ern [34, Wheeler and Yotov [66, and Lovadina and Stenberg [44. For some discussion of these results, we refer to 62. Recently, new works appeared. Repin and Smolianski [54] are able to give a guaranteed upper bound, which may, however, not be sufficiently precise for inhomogeneous $\mathbf{S}$ and general domains and boundary conditions. No local efficiency is shown. Nicaise and Creusé [4] improve the results of [23] and extend them to the anisotropic case. Kim [41] presents estimates applicable to any locally conservative method, as is the case of the estimates presented here. Bounds up to an undetermined constant are given in a mesh-dependent norm, which contains a weighted jump term for the potential. The results of Repin et al. [55] are only valid under the hypothesis that $\mathbf{u}_{h} \in \mathbf{H}(\operatorname{div}, \Omega)$ and $p_{h} \in H_{0}^{1}(\Omega)$, which is not the case for (1.5a)-1.5b) (see also Section 6.4.2 below for further remarks on this point). Larson and Målqvist 43] give energy norm error estimates for the flux. The upper bound again features an unknown constant and no local efficiency is proved. Finally, guaranteed and locally efficient a posteriori error estimates for the lowest-order Raviart-Thomas-Nédélec case with effectivity indices close to the optimal value of one, of the type presented in this paper, were derived in [62, 61] and in Ainsworth [6].

First, in Section 3 of this paper, after collecting some preliminaries in Section 2 , we give an abstract estimate on the energy norm of the difference between two arbitrary vector fields. This estimate will then be used in order to obtain both a priori and a posteriori estimates on the error in the approximation of $\mathbf{u}$ in a straightforward way. In Section 4 we then recall some basic facts about mixed finite element methods and in particular the postprocessing of $[8$ and, for the lowest-order Raviart-Thomas-Nédélec case, that of 62. This postprocessing is the basis for optimal a priori and a posteriori error estimates on the error in the approximation of $p$.

We carry out the a priori error analysis in Section 5. We highlight here its main ideas for the case $\mathbf{S}=\mathbb{I}$ (II denotes the identity matrix). Typically, one has $\left.\mathbf{V}_{h} \cdot \mathbf{n}\right|_{\mathcal{E}_{h}}=\mathbb{P}_{k}\left(\mathcal{E}_{h}\right)$ in mixed finite element methods, where $\mathcal{E}_{h}$ is the set of sides (edges if $d=2$ and faces if $d=3$ ). Our main assumption is that there exists a space $M_{h}$ such that $M_{h}$ is continuous enough in the sense that it is contained in the space of functions such that the jumps of their traces are orthogonal to the polynomials from $\mathbb{P}_{k}\left(\mathcal{E}_{h}\right)$. We also suppose that one can construct a postprocessed potential $\tilde{p}_{h} \in M_{h}$ such that the $\mathbf{L}^{2}(\Omega)$-orthogonal projection of $-\nabla \tilde{p}_{h}$ onto $\Pi_{K \in \mathcal{T}_{h}} \mathbf{V}_{h}(K)$ is $\mathbf{u}_{h}$. This is the situation of the postprocessing of $[8]$. Moreover, recalling that the $L^{2}(\Omega)$-orthogonal projection of $\nabla \cdot \mathbf{u}_{h}$ onto $\Phi_{h}$ equals that of $f$ by $(1.5 \mathrm{~b})$, we note that this fully mimics the continuous setting where $\mathbf{u} \in \mathbf{H}(\operatorname{div}, \Omega), p \in H_{0}^{1}(\Omega)$, and (1.3a) - 1.3b) holds true. Now proving the equivalence between the energy seminorms on $M_{h}(K)$ and the $\mathbf{L}^{2}(K)$-orthogonal projection of $-\nabla M_{h}(K)$ onto 
$\mathbf{V}_{h}(K)$ for each element $K$ enables us to relate the energy error in $p-\tilde{p}_{h}$ to the one in $\mathbf{u}-\mathbf{u}_{h}$, easily obtained itself from the above-mentioned abstract estimate for vector functions. $L^{2}(\Omega)$ estimates then follow by the discrete Friedrichs inequality. We also show that using the postprocessing of 62 in the lowest-order RaviartThomas-Nédélec case, much of the above can be avoided and one obtains the estimates for $p-\tilde{p}_{h}$ in an extremely simple way. Finally, by construction, $p_{h}$ is the $L^{2}(\Omega)$-orthogonal projection of $\tilde{p}_{h}$ onto $\Phi_{h}$, so that the estimates for the error in $p-p_{h}$ are easily recovered. The analysis still relies on the appropriate vector interpolation operator of each mixed finite element method, satisfying the commuting diagram property; see [20, Section III.3]. On the other hand, the use of the uniform-in- $h$ discrete inf-sup condition is avoided by the postprocessing and the discrete Friedrichs inequality; for some related comments on this last point, we refer to [30, 31] and [8, Theorem 2].

In Section 6, we extend the a posteriori error estimates for the lowest-order Raviart-Thomas-Nédélec case of 62] to other families of mixed finite elements, all orders, and grids consisting of rectangular parallelepipeds, using only the techniques that go back to the Prager-Synge equality [51. Using the abstract framework for the error between two arbitrary vector fields of Section 3, we first give estimates for the energy error in the approximation of $\mathbf{u}$. It consists of two parts. The first one is generally given by $\inf _{s \in H_{0}^{1}(\Omega)} \mid\left\|\mathbf{u}_{h}+\mathbf{S} \nabla s\right\|_{*}$, expressing the measure of how close $\mathbf{u}_{h}$ is to the flux of a $H_{0}^{1}(\Omega)$-potential in the vector energy norm $\|\mid \cdot\|_{*}$. In practice, the indicator of an element $K$ is given by $\left\|\left|\mathbf{u}_{h}+\mathbf{S} \nabla\left(\mathcal{I}_{\text {av }}\left(\tilde{p}_{h}\right)\right)\right|\right\|_{*, K}$, where $\mathcal{I}_{\text {av }}$ is an averaging operator. The second one is the residual term (sometimes considered separately and called the "data oscillation term"), given by $C_{\mathrm{P}}^{1 / 2} h_{K} c_{\mathbf{S}, K}^{-1 / 2}\left\|f-P_{\Phi_{h}}(f)\right\|_{K}$, where $h_{K}$ is the diameter of $K, c_{\mathbf{S}, K}$ is the smallest eigenvalue of $\mathbf{S}$ on $K, C_{\mathrm{P}}=1 / \pi^{2}$ is the constant from the Poincaré inequality, $P_{\Phi_{h}}$ is the $L^{2}(\Omega)$-orthogonal projection onto $\Phi_{h}$, and $\|\cdot\|$ is the $L^{2}$ norm. Such an estimator, in particular, improves on estimators of the type $h_{K}\left\|\mathbf{u}_{h}+\mathbf{S} \nabla p_{h}\right\|_{K}$, found in many of the above-cited works. Note that this last estimator, in particular, reduces to $h_{K}\left\|\mathbf{u}_{h}\right\|_{K}$ in low-order mixed finite element methods, i.e., the weighted $L^{2}(\Omega)$-norm of the approximate flux, where no approximation is reflected. Next, using the framework introduced in [62] and [41, we give estimates for the energy error in the approximation of $p$.

The a posteriori error estimates developed in this paper are quite general and apply directly to any locally conservative method, such as the finite volume one; cf. Eymard et al. [36, Aavatsmark et al. 11, or Droniou and Eymard [32, mimetic finite difference; cf. Brezzi et al. 21, covolume; cf. Chou et al. 27, and others. For related results, we refer to [64. They are given for a general diffusion tensor, require no additional regularity of the weak solution, no saturation assumption, and no use of the Helmholtz decomposition. They allow for grids consisting of rectangular parallelepipeds, which can be very useful in practice, where such grids are extensively used. Combinations of simplices and rectangular parallelepipeds in one grid and extensions to nonmatching grids, along with other extensions, are considered in [50. Homogeneous Dirichlet boundary conditions are only considered for the simplicity of the exposition; for inhomogeneous Dirichlet/Neumann boundary conditions, we refer, e.g., to. [1, 64. Numerical experiments in the lowest-order case are presented in 62 .

Finally, in Section 7 we give some complements on mixed finite element methods. In particular, we show that under certain conditions, the weak solution $p$ is 
the orthogonal projection of the postprocessed mixed finite element approximation $\tilde{p}_{h}$ onto the $H_{0}^{1}(\Omega)$ space. This stands in parallel and simultaneously in converse to Galerkin finite element methods, where the approximate solution is the orthogonal projection of the weak solution onto the discrete space. We also show that mixed finite element approximations have close relations to some generalized weak solutions, independently of the smoothness of the tensor $\mathbf{S}$.

\section{Preliminaries}

We set up in this section the notation for meshes and functional spaces used throughout the paper, define scalar- and vector-valued bilinear forms and energy (semi-)norms, and describe an averaging operator.

2.1. Notation. We shall work in this paper with triangulations $\mathcal{T}_{h}$ which for all $h>0$ consist either of closed simplices or of closed rectangular parallelepipeds $K$ such that $\bar{\Omega}=\bigcup_{K \in \mathcal{T}_{h}} K$. We suppose that $\mathcal{T}_{h}$ are conforming (matching), i.e., such that if $K, L \in \mathcal{T}_{h}, K \neq L$, then $K \cap L$ is either an empty set or a common face, edge, or vertex of $K$ and $L$. Let $h_{K}$ denote the diameter of $K$ and let $h:=\max _{K \in \mathcal{T}_{h}} h_{K}$. We denote by $\mathcal{E}_{h}$ the set of all sides of $\mathcal{T}_{h}$, by $\mathcal{E}_{h}^{\text {int }}$ the set of interior, by $\mathcal{E}_{h}^{\text {ext }}$ the set of boundary, and by $\mathcal{E}_{K}$ the set of all the sides of an element $K \in \mathcal{T}_{h} ; h_{\sigma}$ then stands for the diameter of $\sigma \in \mathcal{E}_{h}$. We will also use the notation $\mathcal{T}_{K}\left(\tilde{\mathcal{E}}_{K}\right.$, respectively) for such $L \in \mathcal{T}_{h}\left(\sigma \in \mathcal{E}_{h}\right)$ which share at least a vertex with a $K \in \mathcal{T}_{h}$. Similarly, $\mathcal{T}_{V}$ is the set of such $K \in \mathcal{T}_{h}$ that contain the node $V$. Later on, we will sometimes need the assumption that $\mathcal{T}_{h}$ are shape-regular in the sense that there exists a constant $\kappa_{\mathcal{T}}>0$ such that $\max _{K \in \mathcal{T}_{h}} \kappa_{K} \leq \kappa_{\mathcal{T}}$ for all $h>0$, where $\kappa_{K}:=h_{K} / \varrho_{K}$ with $\varrho_{K}$ being the diameter of the largest ball inscribed in $K$.

Next, for $K \in \mathcal{T}_{h}, \mathbf{n}$ will always denote its exterior normal vector; we shall also employ the notation $\mathbf{n}_{\sigma}$ for a normal vector of a side $\sigma \in \mathcal{E}_{h}$, whose orientation is chosen arbitrarily but fixed for interior sides and coinciding with the exterior normal of $\Omega$ for boundary sides. For $\sigma \in \mathcal{E}_{h}^{\text {int }}$ shared by $K, L \in \mathcal{T}_{h}$ such that $\mathbf{n}_{\sigma}$ points from $K$ to $L$ and a function $\varphi \in H^{1}\left(\mathcal{T}_{h}\right)$ (see below for the notation), we shall define the jump operator $\llbracket \cdot \rrbracket$ by

$$
\llbracket \varphi \rrbracket:=\left.\left(\left.\varphi\right|_{K}\right)\right|_{\sigma}-\left.\left(\left.\varphi\right|_{L}\right)\right|_{\sigma} .
$$

We put $\llbracket \varphi \rrbracket_{\sigma}:=\left.\varphi\right|_{\sigma}$ for any $\sigma \in \mathcal{E}_{h}^{\text {ext }}$.

For a given domain $S \subset \mathbb{R}^{d}$, we shall hereafter employ the standard functional notations $L^{2}(S), H^{q}(S), H_{0}^{1}(S)$; cf. [4. In particular, we note by $(\cdot, \cdot)_{S}$ the $L^{2}(S)$ inner product, by $\|\cdot\|_{S}$ the associated norm (we omit the index $S$ when $S=\Omega$ ), and by $|S|$ the Lebesgue measure of $S$. Let next $\mathbf{H}(\operatorname{div}, S)=\left\{\mathbf{v} \in \mathbf{L}^{2}(S) ; \nabla \cdot \mathbf{v} \in L^{2}(S)\right\}$ and let $\langle\cdot, \cdot\rangle_{\partial S}$ stand for the $(d-1)$-dimensional $L^{2}(\partial S)$-inner product on $\partial S$ or the appropriate duality pairing on $\partial S$. We will also need the space $\tilde{\mathbf{H}}(\operatorname{div}, S)=\{\mathbf{v} \in$ $\left.\mathbf{L}^{q}(S) ; \nabla \cdot \mathbf{v} \in L^{2}(S)\right\}, q>2$ fixed; cf. [20, Section III.3.3]. For a given partition $\mathcal{T}_{h}$ of $\Omega$, let $H^{1}\left(\mathcal{T}_{h}\right):=\left\{\varphi \in L^{2}(\Omega) ;\left.\varphi\right|_{K} \in H^{1}(K) \forall K \in \mathcal{T}_{h}\right\}$ be the broken Sobolev space. Also, we let $W_{0}\left(\mathcal{T}_{h}\right)$ and $W_{h}\left(\mathcal{T}_{h}\right)$ be the spaces of functions with jumps of traces across the sides orthogonal to, respectively, constants and polynomials of $\left.\mathbf{V}_{h} \cdot \mathbf{n}\right|_{\sigma}$ for each $\sigma \in \mathcal{E}_{h}$,

$$
\begin{array}{lll}
W_{0}\left(\mathcal{T}_{h}\right):=\left\{\varphi \in H^{1}\left(\mathcal{T}_{h}\right) ;\langle\llbracket \varphi \rrbracket, 1\rangle_{\sigma}=0\right. & \left.\forall \sigma \in \mathcal{E}_{h}\right\}, \\
W_{h}\left(\mathcal{T}_{h}\right):=\left\{\varphi \in H^{1}\left(\mathcal{T}_{h}\right) ;\left\langle\llbracket \varphi \rrbracket, \psi_{h}\right\rangle_{\sigma}=0\right. & \left.\forall \psi_{h} \in \mathbf{V}_{h} \cdot \mathbf{n}\right|_{\sigma} & \left.\forall \sigma \in \mathcal{E}_{h}\right\}
\end{array}
$$


Clearly, $W_{0}\left(\mathcal{T}_{h}\right), W_{h}\left(\mathcal{T}_{h}\right) \not \subset H_{0}^{1}(\Omega)$ but there is "less and less nonconformity" in $W_{h}\left(\mathcal{T}_{h}\right)$ with increasing order of the polynomials in $\mathbf{V}_{h} \cdot \mathbf{n}$. Finally, the weak gradient on $H^{1}(\Omega)$ and the piecewise weak gradient on $H^{1}\left(\mathcal{T}_{h}\right)$ are both denoted by the $\nabla$ sign and similarly for the weak divergence $\nabla \cdot$. To simplify the notation, we systematically use the convention $0 / 0=0$ throughout the text.

Finally, we denote by $c_{\mathbf{S}, \Omega}, C_{\mathbf{S}, \Omega}$ the best constants such that $c_{\mathbf{S}, \Omega} \mathbf{v} \cdot \mathbf{v} \leq \mathbf{S v} \cdot \mathbf{v} \leq$ $C_{\mathbf{S}, \Omega} \mathbf{v} \cdot \mathbf{v}, c_{\mathbf{S}, \Omega}>0, C_{\mathbf{S}, \Omega}>0$, for all $\mathbf{v} \in \mathbb{R}^{d}$ and a.e. in $\Omega$. Similar notations $c_{\mathbf{S}, K}$, $C_{\mathbf{S}, K}$, and $c_{\mathbf{S}, \mathcal{T}_{K}}$ for $K \in \mathcal{T}_{h}$ will also be employed.

2.2. Bilinear forms and energy (semi-)norms. Let the symmetric bilinear form $\mathcal{B}$ acting on scalars be defined by

$$
\mathcal{B}(p, \varphi):=(\mathbf{S} \nabla p, \nabla \varphi), \quad p, \varphi \in H^{1}\left(\mathcal{T}_{h}\right),
$$

whereas its vector counterpart $\mathcal{A}$ acting on vectors is defined by

$$
\mathcal{A}(\mathbf{u}, \mathbf{v}):=\left(\mathbf{u}, \mathbf{S}^{-1} \mathbf{v}\right), \quad \mathbf{u}, \mathbf{v} \in \mathbf{L}^{2}(\Omega) .
$$

Note that the primal weak formulation (1.2) can be rewritten equivalently using the above forms $\mathcal{B}$ and $\mathcal{A}$ as: find $p \in H_{0}^{1}(\Omega)$ such that

$$
\mathcal{B}(p, \varphi)=(f, \varphi) \quad \forall \varphi \in H_{0}^{1}(\Omega)
$$

or

$$
\mathcal{A}(\mathbf{S} \nabla p, \mathbf{S} \nabla \varphi)=(f, \varphi) \quad \forall \varphi \in H_{0}^{1}(\Omega),
$$

as

$$
\mathcal{B}(p, \varphi)=\mathcal{A}(\mathbf{S} \nabla p, \mathbf{S} \nabla \varphi) \quad \forall p, \varphi \in H^{1}\left(\mathcal{T}_{h}\right),
$$

which will turn out to be useful later. Let us define the energy seminorm on the space $H^{1}\left(\mathcal{T}_{h}\right)$,

$$
\|\varphi\|^{2}:=\mathcal{B}(\varphi, \varphi)=\left\|\mathbf{S}^{\frac{1}{2}} \nabla \varphi\right\|^{2}, \quad \varphi \in H^{1}\left(\mathcal{T}_{h}\right),
$$

which becomes a norm on $W_{0}\left(\mathcal{T}_{h}\right)$ thanks to the discrete Friedrichs inequality

$$
\|\varphi\|_{\Omega} \leq C_{\mathrm{DF}}^{\frac{1}{2}}\|\nabla \varphi\| \quad \forall \varphi \in W_{0}\left(\mathcal{T}_{h}\right), \forall h>0,
$$

where $C_{\mathrm{DF}}$ only depends on $\kappa_{\mathcal{T}}$ and $\inf _{\mathbf{b} \in \mathbb{R}^{d}}\left\{\operatorname{thick}_{\mathbf{b}}(\Omega)\right\}$; cf. [59, Theorem 5.4]. Similarly, let the energy norm for vectors be given by

$$
\|\mathbf{v}\|_{*}^{2}:=\mathcal{A}(\mathbf{v}, \mathbf{v})=\left\|\mathbf{S}^{-\frac{1}{2}} \mathbf{v}\right\|^{2}, \quad \mathbf{v} \in \mathbf{L}^{2}(\Omega) .
$$

Note in particular that by (2.6),

$$
\|\varphi\|\|=\| \mathbf{S} \nabla \varphi \|_{*} \quad \forall \varphi \in H^{1}\left(\mathcal{T}_{h}\right) .
$$

By the Cauchy-Schwarz inequality, one also immediately has

$$
\begin{array}{ll}
\mathcal{B}(p, \varphi) \leq|\|p|\||\|\varphi \mid\| & \forall p, \varphi \in H^{1}\left(\mathcal{T}_{h}\right), \\
\mathcal{A}(\mathbf{u}, \mathbf{v}) \leq\left|\left\|\mathbf { u } \left|\left\|_{*} \mid\right\| \mathbf{v} \|_{*}\right.\right.\right. & \forall \mathbf{u}, \mathbf{v} \in \mathbf{L}^{2}(\Omega) .
\end{array}
$$

We will also use the "div-energy" norm for vectors, defined as

$$
\|\mathbf{v}\|\left\|_{*, \operatorname{div}}^{2}:=\right\| \mathbf{v}\left\|_{*}^{2}+\right\| \nabla \cdot \mathbf{v} \|^{2}, \quad \mathbf{v} \in \mathbf{H}(\operatorname{div}, \Omega) .
$$

Let us finally recall that, for $K \in \mathcal{T}_{h}$, the Poincaré inequality states that

$$
\left\|\varphi-\pi_{0}(\varphi)\right\|_{K}^{2} \leq C_{\mathrm{P}} h_{K}^{2}\|\nabla \varphi\|_{K}^{2} \quad \forall \varphi \in H^{1}(K),
$$


where $\pi_{l}$ denotes the $L^{2}(\Omega)$-orthogonal projection onto piecewise polynomials of degree $l$. Thanks to the convexity of simplices and rectangular parallelepipeds, $C_{\mathrm{P}}=1 / \pi^{2}$; cf. [49, 13].

2.3. An averaging operator. We shall work later with piecewise polynomial approximations $\tilde{p}_{h}$ to $p$, nonconforming in the sense that $\tilde{p}_{h} \notin H_{0}^{1}(\Omega)$ but satisfying $\tilde{p}_{h} \in W_{h}\left(\mathcal{T}_{h}\right)\left(\tilde{p}_{h} \in H^{1}\left(\mathcal{T}_{h}\right)\right.$ in general). It will also turn out that we will need their conforming (continuous, contained in $H_{0}^{1}(\Omega)$ ) interpolant. We will use for this purpose the averaging operator previously considered in, e.g., in 33, 3, 40, 5, 35. and analyzed in detail in [40, 22]. This operator is sometimes called Oswald. Note that the averaging procedure is applied here to the potential and not to its gradient as in 69 .

If $\mathcal{T}_{h}$ consist of simplices, let $\mathbb{R}_{n}\left(\mathcal{T}_{h}\right):=\mathbb{P}_{n}\left(\mathcal{T}_{h}\right)$ denote the space of piecewise polynomials of total degree at most $n$ on each simplex (without any continuity requirement on the sides). Similarly, if $\mathcal{T}_{h}$ consist of rectangular parallelepipeds, let $\mathbb{R}_{n}\left(\mathcal{T}_{h}\right):=\mathbb{Q}_{n}\left(\mathcal{T}_{h}\right)$ denote the space of piecewise polynomials of degree at most $n$ in each variable. The averaging operator $\mathcal{I}_{\text {av }}: \mathbb{R}_{n}\left(\mathcal{T}_{h}\right) \rightarrow \mathbb{R}_{n}\left(\mathcal{T}_{h}\right) \cap H_{0}^{1}(\Omega)$ is defined as follows: given a function $\varphi_{h} \in \mathbb{R}_{n}\left(\mathcal{T}_{h}\right)$, the value of $\mathcal{I}_{\text {av }}\left(\varphi_{h}\right)$ is prescribed at the Gauss-Lobatto nodes on rectangular parallelepipeds and Lagrangian nodes on simplices of $\mathbb{R}_{n}\left(\mathcal{T}_{h}\right) \cap H_{0}^{1}(\Omega)$ by the average of the values of $\varphi_{h}$ at this node,

$$
\mathcal{I}_{\mathrm{av}}\left(\varphi_{h}\right)(V)=\left.\frac{1}{\left|\mathcal{T}_{V}\right|} \sum_{K \in \mathcal{T}_{V}} \varphi_{h}\right|_{K}(V)
$$

where $\left|\mathcal{T}_{V}\right|$ stands for the cardinality of $\mathcal{T}_{V}$. Note that the interpolant is in particular equal to $\left.\varphi_{h}\right|_{K}(V)$ at a node $V$ lying in the interior of some $K \in \mathcal{T}_{h}$. At boundary nodes, the value of $\mathcal{I}_{\text {av }}\left(\varphi_{h}\right)$ is set to zero. The following results have been proved in [22, Lemmas 3.2 and 5.3 and Remark 3.2] and [40, Theorem 2.2]:

Lemma 2.1 (Averaging operator). Let $\mathcal{T}_{h}$ be shape-regular, let $\varphi_{h} \in \mathbb{R}_{n}\left(\mathcal{T}_{h}\right)$, and let $\mathcal{I}_{\mathrm{av}}\left(\varphi_{h}\right)$ be constructed as described above. Then

$$
\left\|\nabla\left(\varphi_{h}-\mathcal{I}_{\text {av }}\left(\varphi_{h}\right)\right)\right\|_{K}^{2} \leq C \sum_{\sigma \in \tilde{\mathcal{E}}_{K}} h_{\sigma}^{-1}\left\|\llbracket \varphi_{h} \rrbracket\right\|_{\sigma}^{2}
$$

for all $K \in \mathcal{T}_{h}$, where the constant $C$ depends only on the space dimension $d$, on the maximal polynomial degree $n$, and on the shape regularity parameter $\kappa_{\mathcal{T}}$.

\section{Abstract framework}

We develop in the first part of this section an abstract estimate on the energy norm of the difference between two arbitrary vector fields which will enable us to easily carry out both the a priori and a posteriori error analysis of mixed finite element methods in a unified way. In the second part of this section, we give a slightly improved version of the estimate, suitable for a posteriori error estimation.

3.1. A general abstract estimate. Following the approach introduced in 62 , Lemma 7.1], we have the following abstract result:

Theorem 3.1 (General abstract estimate). Let $\mathbf{v}, \mathbf{w}, \mathbf{t} \in \mathbf{L}^{2}(\Omega)$ be arbitrary. Then

$$
\left\|\mathbf{v}-\mathbf{w}\left|\left\|_{*} \leq\right\|\right| \mathbf{w}-\mathbf{t}\right\|_{*}+\left|\mathcal{A}\left(\mathbf{v}-\mathbf{w}, \frac{\mathbf{v}-\mathbf{t}}{\|\mathbf{v}-\mathbf{t} \mid\|_{*}}\right)\right| .
$$


Proof. Let us first suppose that $\||\mathbf{v}-\mathbf{w}|\|_{*} \leq\||\mathbf{v}-\mathbf{t}|\|_{*}$. We then have

$$
\begin{aligned}
\|\mathbf{v}-\mathbf{t} \mid\|_{*}^{2} & =\mathcal{A}(\mathbf{v}-\mathbf{t}, \mathbf{v}-\mathbf{t})=\mathcal{A}(\mathbf{v}-\mathbf{w}, \mathbf{v}-\mathbf{t})+\mathcal{A}(\mathbf{w}-\mathbf{t}, \mathbf{v}-\mathbf{t}) \\
& \leq\|\mathbf{v}-\mathbf{t}\|\left\|_{*} \mathcal{A}\left(\mathbf{v}-\mathbf{w}, \frac{\mathbf{v}-\mathbf{t}}{\|\mathbf{v}-\mathbf{t}\|_{*}}\right)+\right\||\mathbf{w}-\mathbf{t}|\left\|_{*}\right\| \mathbf{v}-\mathbf{t} \mid \|_{*},
\end{aligned}
$$

using the bilinearity of $\mathcal{A}(\cdot, \cdot),(2.9)$, and (2.11b). In view of the assumption, this finishes the proof in the first case.

If $\|\mathbf{v}-\mathbf{t}\|\left\|_{*} \leq\right\| \mathbf{v}-\mathbf{w} \|_{*}$ holds, then

$$
\begin{aligned}
\|\| \mathbf{v}-\mathbf{w} \|_{*}^{2} & =\mathcal{A}(\mathbf{v}-\mathbf{w}, \mathbf{v}-\mathbf{w})=\mathcal{A}(\mathbf{v}-\mathbf{w}, \mathbf{v}-\mathbf{t})+\mathcal{A}(\mathbf{v}-\mathbf{w}, \mathbf{t}-\mathbf{w}) \\
& \leq\left|\left\|\mathbf{v}-\mathbf{t}\left|\left\|_{*} \mathcal{A}\left(\mathbf{v}-\mathbf{w}, \frac{\mathbf{v}-\mathbf{t}}{\|\mathbf{v}-\mathbf{t}\|_{*}}\right)+\right\| \mathbf{v}-\mathbf{w}\right|\right\|_{*}\|\mathbf{w}-\mathbf{t} \mid\|_{*}\right. \\
& \leq\left|\left\|\mathbf{v}-\mathbf{w}\left|\left\|_{*}\left|\mathcal{A}\left(\mathbf{v}-\mathbf{w}, \frac{\mathbf{v}-\mathbf{t}}{\|\mathbf{v}-\mathbf{t} \mid\|_{*}}\right)\right|+\right\| \mathbf{v}-\mathbf{w}\right|\right\|_{*}\|\mid \mathbf{w}-\mathbf{t}\|_{*},\right.
\end{aligned}
$$

whence again the assertion follows. Thus the proof is complete.

Remark 3.2 (General abstract estimate). Using the triangle inequality, the bilinearity of $\mathcal{A}(\cdot, \cdot)$, and (2.11b), we immediately have

$$
\begin{aligned}
\|\mathbf{v}-\mathbf{w}\|_{*} & \leq\|\mathbf{w}-\mathbf{t}\|\left\|_{*}+\left|\|\mathbf{v}-\mathbf{t} \mid\|_{*}=\|\mathbf{w}-\mathbf{t}\|_{*}+\mathcal{A}\left(\mathbf{v}-\mathbf{t}, \frac{\mathbf{v}-\mathbf{t}}{\|\mathbf{v}-\mathbf{t}\| \|_{*}}\right)\right.\right. \\
& \leq\|\mathbf{w}-\mathbf{t}\|_{*}+\mathcal{A}\left(\mathbf{v}-\mathbf{w}, \frac{\mathbf{v}-\mathbf{t}}{\|\mathbf{v}-\mathbf{t}\|_{*}}\right)+\mathcal{A}\left(\mathbf{w}-\mathbf{t}, \frac{\mathbf{v}-\mathbf{t}}{\|\mathbf{v}-\mathbf{t}\|_{*}}\right) \\
& \leq 2\|\mathbf{w}-\mathbf{t}\|_{*}+\mathcal{A}\left(\mathbf{v}-\mathbf{w}, \frac{\mathbf{v}-\mathbf{t}}{\|\mathbf{v}-\mathbf{t}\|_{*}}\right) .
\end{aligned}
$$

The estimate of Theorem 3.1 is superior to this simple bound by removing the factor 2 at the term $\|\mathbf{w}-\mathbf{t}\|_{*}$. In comparison to Theorem 3.3 below, the advantage of Theorem 3.1 is that any triple of functions from $\mathbf{L}^{2}(\Omega)$ can be chosen. Moreover, it turns out that it is extensible to the convection-diffusion-reaction framework, where it, in addition, shows advantageously that $\mathbf{t} \in \mathbf{L}^{2}(\Omega)$ in the second argument of $\mathcal{A}(\cdot, \cdot)$ can be chosen arbitrarily; cf. [62].

3.2. A Pythagorean estimate. Following the approach introduced in Kim 41, Lemma 4.4], we have the following estimate:

Theorem 3.3 (Pythagorean abstract estimate). Let $\mathbf{v}$ be such that $\mathbf{v}=-\mathbf{S} \nabla \vartheta$ for some $\vartheta \in H_{0}^{1}(\Omega)$ and let $\mathbf{w} \in \mathbf{L}^{2}(\Omega)$ be arbitrary. Next, let $\psi \in H_{0}^{1}(\Omega)$ be the solution of the problem

$$
\mathcal{B}(\psi, \varphi)=\mathcal{A}(-\mathbf{w}, \mathbf{S} \nabla \varphi) \quad \forall \varphi \in H_{0}^{1}(\Omega) .
$$

Then

$$
\||\mathbf{v}-\mathbf{w}|\|_{*}^{2}=\left|\|\mathbf{w}+\mathbf{S} \nabla \psi \mid\|_{*}^{2}+\mathcal{A}\left(\mathbf{v}-\mathbf{w}, \frac{\mathbf{v}+\mathbf{S} \nabla \psi}{\|\mathbf{v}+\mathbf{S} \nabla \psi\|_{*}}\right)^{2} .\right.
$$

Moreover,

$$
\left\|\mathbf{w}+\mathbf{S} \nabla \psi\left|\left\|_{*}=\inf _{s \in H_{0}^{1}(\Omega)}\left|\|\mathbf{w}+\mathbf{S} \nabla s \mid\|_{*}\right.\right.\right.\right.
$$


Proof. Let us first note that there exists a unique solution to the problem (3.1) by the Riesz representation theorem, as $\mathcal{A}(\mathbf{w},-\mathbf{S} \nabla(\cdot))$ is a continuous linear form. Note as well that (3.1) can be equivalently written, using (2.6), as

$$
\mathcal{A}(\mathbf{S} \nabla \psi+\mathbf{w}, \mathbf{S} \nabla \varphi)=0 \quad \forall \varphi \in H_{0}^{1}(\Omega) .
$$

Using this characterization for $\varphi=\psi-\vartheta$, we have

$$
\begin{aligned}
\|\mathbf{v}-\mathbf{w}\|_{*}^{2}= & \mathcal{A}(\mathbf{v}-\mathbf{w}, \mathbf{v}-\mathbf{w})=\mathcal{A}(\mathbf{v}-\mathbf{w}, \mathbf{v}+\mathbf{S} \nabla \psi)+\mathcal{A}(\mathbf{v}-\mathbf{w},-\mathbf{S} \nabla \psi-\mathbf{w}) \\
= & \mathcal{A}(\mathbf{v}+\mathbf{S} \nabla \psi, \mathbf{v}+\mathbf{S} \nabla \psi)-2 \mathcal{A}(\mathbf{S} \nabla \psi+\mathbf{w}, \mathbf{v}+\mathbf{S} \nabla \psi) \\
& +\mathcal{A}(\mathbf{w}+\mathbf{S} \nabla \psi, \mathbf{w}+\mathbf{S} \nabla \psi) \\
= & \|\mathbf{v}+\mathbf{S} \nabla \psi\|_{*}^{2}+\|\mathbf{w}+\mathbf{S} \nabla \psi\|_{*}^{2},
\end{aligned}
$$

employing also the definition and the symmetry of $\mathcal{A}(\cdot, \cdot)$. The proof is finished by noticing that, by (3.4),

$$
\|\mathbf{v}+\mathbf{S} \nabla \psi\|_{*}=\mathcal{A}\left(\mathbf{v}-\mathbf{w}, \frac{\mathbf{v}+\mathbf{S} \nabla \psi}{\|\mathbf{v}+\mathbf{S} \nabla \psi\|_{*}}\right)
$$

and

$$
\|\mathbf{w}+\mathbf{S} \nabla \psi\|_{*}^{2}=\mathcal{A}(\mathbf{w}+\mathbf{S} \nabla s, \mathbf{w}+\mathbf{S} \nabla \psi) \leq\|\mathbf{w}+\mathbf{S} \nabla s\|\left\|_{*}\right\| \mathbf{w}+\mathbf{S} \nabla \psi \|_{*}
$$

for an arbitrary $s \in H_{0}^{1}(\Omega)$, whence (3.3) follows.

This Pythagorean estimate, as we will see later, gives a slightly more precise upper bound in a posteriori error estimates.

\section{The MiXed Finite ELEMENT METHOD}

We recall here some known basic facts about the mixed finite element method, namely the existence and uniqueness of discrete solutions, hybridization, and, most importantly, the postprocessing of [8]. We start by giving examples of the most common mixed finite element spaces.

4.1. Examples of local mixed finite element spaces. Table 1 lists the most common mixed finite element spaces $\mathbf{V}_{h}(K) \times \Phi_{h}(K)$ on an element $K \in \mathcal{T}_{h}$. The notation RTN stands for the Raviart-Thomas 53 , space on triangles and rectangles and the Nédélec [47] space on tetrahedra and rectangular parallelepipeds if $d=3$ and BDM for the Brezzi-Douglas-Marini [19] space on triangles and rectangles and the Brezzi-Douglas-Durán-Fortin [18] space on tetrahedra and rectangular parallelepipeds if $d=3$. In the notation, "s" stands for simplices, "r" for rectangular parallelepipeds, $\mathbf{P}_{2, k}^{*}:=r \nabla \times\left(x^{k+1} y\right)+s \nabla \times\left(x y^{k+1}\right), r, s \in \mathbb{R}$, and $\mathbf{P}_{3, k}^{*}:=\sum_{i=0}^{k}\left\{r_{i} \nabla \times\right.$ $\left.\left(0,0, x y^{i+1} z^{k-i}\right)^{t}+s_{i} \nabla \times\left(x^{k-i} y z^{i+1}, 0,0\right)^{t}+t_{i} \nabla \times\left(0, x^{i+1} y^{k-i} z, 0\right)^{t}\right\}, r_{i}, s_{i} \in \mathbb{R}$, with $\nabla \times$ the curl operator. Here we have denoted by $k$ the biggest polynomial space contained in $\mathbf{V}_{h}(K)$ and by $l$ that in $\Phi_{h}(K)$. Then $\mathbf{V}_{h}:=\Pi_{K \in \mathcal{T}_{h}} \mathbf{V}_{h}(K) \cap \mathbf{H}(\operatorname{div}, \Omega)$ and $\Phi_{h}:=\Pi_{K \in \mathcal{T}_{h}} \Phi_{h}(K)$. Note in particular that whereas $\mathbf{V}_{h}(K)$ are local unconstrained spaces, the fact that $\mathbf{V}_{h} \subset \mathbf{H}(\operatorname{div}, \Omega)$ imposes the normal trace continuity of all $\mathbf{v}_{h} \in \mathbf{V}_{h}$, i.e., $\left.\mathbf{v}_{h}\right|_{K} \cdot \mathbf{n}_{\sigma_{K, L}}=\left.\mathbf{v}_{h}\right|_{L} \cdot \mathbf{n}_{\sigma_{K, L}}$ for all $\sigma_{K, L} \in \mathcal{E}_{h}^{\text {int }}$ shared by elements $K$ and $L$. For a general reference to mixed finite element methods, we refer to Brezzi and Fortin [20] or Roberts and Thomas [56].

In the rest of the paper, we shall sometimes consider separately the following particular case: 
TABLE 1. Examples of local mixed finite element spaces

\begin{tabular}{cccccccc}
\hline Space & $d$ & element & $\mathbf{V}_{h}(K)$ & $\Phi_{h}(K)$ & $\left.\mathbf{V}_{h}(K) \cdot \mathbf{n}\right|_{\sigma}$ & $k$ & $l$ \\
\hline $\mathbf{R T N}_{k}^{\mathrm{s}}(K)$ & 2,3 & simplex & $\mathbb{P}_{k}^{d}+\mathbb{P}_{k} \mathbf{x}$ & $\mathbb{P}_{k}$ & $\mathbb{P}_{k}$ & $\geq 0$ & $=k$ \\
$\mathbf{R T N}_{k}^{\mathrm{r}}(K)$ & 2,3 & rect. par. & $\mathbb{Q}_{k}^{d}+\mathbb{Q}_{k} \mathbf{x}$ & $\mathbb{Q}_{k}$ & $\mathbb{P}_{k}(d=2)$ & $\geq 0$ & $=k$ \\
& & & & & $\mathbb{Q}_{k}(d=3)$ & & \\
$\mathbf{B D M}_{k}^{\mathrm{s}}(K)$ & 2,3 & simplex & $\mathbb{P}_{k}^{d}$ & $\mathbb{P}_{k-1}$ & $\mathbb{P}_{k}$ & $\geq 1=k-1$ \\
$\mathbf{B D M}_{k}^{\mathrm{r}}(K)$ & 2,3 & rect. par. & $\mathbb{P}_{k}^{d} \oplus \mathbf{P}_{d, k}^{*}$ & $\mathbb{P}_{k-1}$ & $\mathbb{P}_{k}$ & $\geq 1=k-1$ \\
\hline
\end{tabular}

Assumption (A) (Lowest-order Raviart-Thomas-Nédélec case). The spaces $\mathbf{V}_{h}$ and $\Phi_{h}$ are formed by $\mathbf{R} \mathbf{T} \mathbf{N}_{0}^{\mathrm{s}}(K)$ or $\mathbf{R} \mathbf{T} \mathbf{N}_{0}^{\mathrm{r}}(K)$ from Table 1 and the tensor $\mathbf{S}$ is piecewise constant on simplices and piecewise constant and diagonal on rectangular parallelepipeds.

4.2. Existence and uniqueness of the discrete solutions. For the sake of completeness and also to stress its simplicity, we recall here the proof of existence and uniqueness of the discrete mixed finite element solution.

Corollary 4.1 (Existence and uniqueness of the discrete mixed finite element solution). Let $\nabla \cdot \mathbf{V}_{h}=\Phi_{h}$. Then there exists a unique solution to the problem (1.5a)(1.5b).

Proof. Problem (1.5a -1.5b) is a square linear finite-dimensional system. It thus suffices to prove that $f=0$ implies $\mathbf{u}_{h}=0$ and $p_{h}=0$. Put $\phi_{h}=p_{h}$ in (1.5b) and $\mathbf{v}_{h}=\mathbf{u}_{h}$ in (1.5a) and sum the equations. This gives $\left(\mathbf{S}^{-1} \mathbf{u}_{h}, \mathbf{u}_{h}\right)=0$, whence $\mathbf{u}_{h}=0$ follows. Consequently, $\left(p_{h}, \nabla \cdot \mathbf{v}_{h}\right)=0$ for all $\mathbf{v}_{h} \in \mathbf{V}_{h}$, whence $p_{h}=0$ follows by the assumption $\nabla \cdot \mathbf{V}_{h}=\Phi_{h}$.

4.3. Hybridization. The hybridization technique allows us to relax the normal trace continuity constraint $\mathbf{V}_{h} \subset \mathbf{H}(\operatorname{div}, \Omega)$ while imposing it instead with the aid of Lagrange multipliers. The unconstrained flux space is given by $\tilde{\mathbf{V}}_{h}:=$ $\Pi_{K \in \mathcal{T}_{h}} \mathbf{V}_{h}(K)$, where $\mathbf{V}_{h}(K)$ are the local spaces on each element, and the Lagrange multipliers space $\Lambda_{h}$ is the space of (discontinuous) piecewise polynomials $\mu_{h}$ on $\mathcal{E}_{h}^{\text {int }}$ such that for all $\sigma \in \mathcal{E}_{h}^{\text {int }},\left.\left.\mu_{h}\right|_{\sigma} \in \mathbf{V}_{h} \cdot \mathbf{n}\right|_{\sigma}$. With these notations, the hybridized version of (1.5a)-1.5b) consists in finding $\mathbf{u}_{h} \in \tilde{\mathbf{V}}_{h}, p_{h} \in \Phi_{h}$, and $\lambda_{h} \in \Lambda_{h}$ such that

$$
\begin{aligned}
\left(\mathbf{S}^{-1} \mathbf{u}_{h}, \mathbf{v}_{h}\right)-\left(p_{h}, \nabla \cdot \mathbf{v}_{h}\right)+\sum_{K \in \mathcal{T}_{h}}\left\langle\mathbf{v}_{h} \cdot \mathbf{n}, \lambda_{h}\right\rangle_{\partial K \backslash \partial \Omega} & =0 & & \forall \mathbf{v}_{h} \in \tilde{\mathbf{V}}_{h}, \\
\left(\nabla \cdot \mathbf{u}_{h}, \phi_{h}\right) & =\left(f, \phi_{h}\right) & & \forall \phi_{h} \in \Phi_{h}, \\
\sum_{K \in \mathcal{T}_{h}}\left\langle\mathbf{u}_{h} \cdot \mathbf{n}, \mu_{h}\right\rangle_{\partial K \backslash \partial \Omega} & =0 & & \forall \mu_{h} \in \Lambda_{h} .
\end{aligned}
$$

It is well known and easy to show that $p_{h}, \mathbf{u}_{h}$ from (1.5a)-1.5b) and (4.1a)-(4.1c) coincide; $\lambda_{h}$ then provides an additional approximation to $p$. Let us also recall that $\lambda_{h}$ can be postprocessed locally from (1.5a)-1.5b); on each $\sigma \in \mathcal{E}_{h}^{\text {int }}, \sigma \in \mathcal{E}_{K}$ for 
some $K \in \mathcal{T}_{h}$, it is given by

$$
\begin{aligned}
\left\langle\mathbf{v}_{h} \cdot \mathbf{n}, \lambda_{h}\right\rangle_{\sigma}=- & \left(\mathbf{S}^{-1} \mathbf{u}_{h}, \mathbf{v}_{h}\right)_{K}+\left(p_{h}, \nabla \cdot \mathbf{v}_{h}\right)_{K} \\
& \forall \mathbf{v}_{h} \in \mathbf{V}_{h}(K) \text { such that }\left.\left(\mathbf{v}_{h} \cdot \mathbf{n}\right)\right|_{\gamma}=0 \forall \gamma \in \mathcal{E}_{K}, \gamma \neq \sigma,
\end{aligned}
$$

so that it is not necessary to implement (4.1a) (4.1c) in order to obtain it.

4.4. Postprocessing. Seemingly, there is no direct analogy of the link $\mathbf{u}=-\mathbf{S} \nabla p$ at the discrete level in the mixed finite element method. It is sometimes even said that the distinctive feature of the mixed finite element method is that the discrete flux $\mathbf{u}_{h}$ has "more regularity" than the discrete potential $p_{h}$, in a sense that it is a polynomial of a higher degree. We shall see in this section that the link $\mathbf{u}_{h} \approx-\mathbf{S} \nabla p_{h}$ can easily be recovered by postprocessing.

Different postprocessing techniques for mixed finite elements have been introduced in the past. Let us cite the works of Arnold and Brezzi [9, Bramble and $\mathrm{Xu}$ [16, Stenberg [57, Chen [25, Arbogast and Chen [8, and, for the lowest-order Raviart-Thomas-Nédélec case, the author 62]. It will turn out that for our purposes, the postprocessing of [62 and [8] under Assumption (A) and that of [8] in general will be optimal. We now recall it here.

4.4.1. Postprocessing in the lowest-order Raviart-Thomas-Nédélec case. Under Assumption (A), the following postprocessing has been proposed in [62, Section 4.1] on simplicial meshes and in [8, Sections 6 and 9] (cf. also [64, Section 3.2]) on meshes consisting of rectangular parallelepipeds: construct $\tilde{p}_{h} \in \mathbb{P}_{2}\left(\mathcal{T}_{h}\right)$ such that

$$
\begin{aligned}
-\left.\mathbf{S}_{K} \nabla \tilde{p}_{h}\right|_{K} & =\left.\mathbf{u}_{h}\right|_{K} & & \forall K \in \mathcal{T}_{h}, \\
\pi_{0}\left(\left.\tilde{p}_{h}\right|_{K}\right) & =\left.p_{h}\right|_{K} & & \forall K \in \mathcal{T}_{h} .
\end{aligned}
$$

Note that $\tilde{p}_{h}$ is in general not a full second-order polynomial and that it is only built on each $K \in \mathcal{T}_{h}$ from the given degrees of freedom, so that its construction cost is negligible.

In general, $\tilde{p}_{h}$ is nonconforming in the sense that $\tilde{p}_{h} \notin H_{0}^{1}(\Omega)$ but it is shown in [62, Lemma 6.1] that $\tilde{p}_{h} \in W_{0}\left(\mathcal{T}_{h}\right)$ on simplicial meshes; for meshes of rectangular parallelepipeds; see 8 . Hence, at least the mean values of $\tilde{p}_{h}$ on the sides of $\mathcal{T}_{h}$ are continuous (and equal to zero on $\partial \Omega$ ). Moreover, these means of traces coincide with the Lagrange multiplies $\lambda_{h}$ of the hybridized version (4.1a) (4.1c) of (1.5a)-(1.5b), see [62, Lemma 6.4] and [8].

4.4.2. Postprocessing in the general case. It turns out that in the general case, there does not exist $\tilde{p}_{h}$ such that (4.2a) is true. Then the postprocessing by Arbogast and Chen [8] proposes a weak form of this relation. This postprocessing is a generalization of the postprocessing proposed originally by Arnold and Brezzi [9] and Chen 25$]$ and it is defined as follows. Let $P_{\Phi_{h}}$ be the $L^{2}(\Omega)$-orthogonal projection onto $\Phi_{h}, P_{\tilde{\mathbf{V}}_{h}}$ the $\mathbf{L}^{2}(\Omega)$-orthogonal projection onto $\tilde{\mathbf{V}}_{h}$ with respect to the scalar 
product $\left(\mathbf{S}^{-1} \cdot, \cdot\right)$, and $P_{\Lambda_{h}}$ the $L^{2}\left(\mathcal{E}_{h}^{\text {int }}\right)$-orthogonal projection onto $\Lambda_{h}$, i.e.,

$P_{\Phi_{h}}: L^{2}(\Omega) \rightarrow \Phi_{h} \quad$ for $\phi \in L^{2}(\Omega), \quad\left(\phi-P_{\Phi_{h}}(\phi), \phi_{h}\right)=0 \quad \forall \phi_{h} \in \Phi_{h}$,

$P_{\tilde{\mathbf{V}}_{h}}: \mathbf{L}^{2}(\Omega) \rightarrow \tilde{\mathbf{V}}_{h} \quad$ for $\mathbf{v} \in \mathbf{L}^{2}(\Omega), \quad\left(\mathbf{S}^{-1}\left(\mathbf{v}-P_{\tilde{\mathbf{V}}_{h}}(\mathbf{v})\right), \mathbf{v}_{h}\right)=0 \quad \forall \mathbf{v}_{h} \in \tilde{\mathbf{V}}_{h}$, (4.3c)

$P_{\Lambda_{h}}: L^{2}\left(\mathcal{E}_{h}^{\text {int }}\right) \rightarrow \Lambda_{h} \quad$ for $\mu \in L^{2}\left(\mathcal{E}_{h}^{\text {int }}\right), \quad\left(\mu-P_{\Lambda_{h}}(\mu), \mu_{h}\right)_{\mathcal{E}_{h}^{\text {int }}}=0 \quad \forall \mu_{h} \in \Lambda_{h}$

Note that these projections are defined locally, as the spaces $\Phi_{h}, \tilde{\mathbf{V}}_{h}$, and $\Lambda_{h}$ do not have any global coupling. The postprocessed potential $\tilde{p}_{h} \in M_{h}$ (the space $M_{h}$ is described below) is then defined by

$$
\begin{aligned}
& P_{\Phi_{h}}\left(\tilde{p}_{h}\right)=p_{h}, \\
& P_{\Lambda_{h}}\left(\tilde{p}_{h}\right)=\lambda_{h} .
\end{aligned}
$$

Note that employing (4.4a) 4.4b) in (4.1a) and using $\nabla \cdot \mathbf{V}_{h}(K)=\Phi_{h}(K)$ and $\left.\mathbf{V}_{h}(K) \cdot \mathbf{n}\right|_{\partial K \backslash \partial \Omega}=\Lambda_{h}(K)$ gives, for all $K \in \mathcal{T}_{h}$,

$$
\left(\mathbf{S}^{-1} \mathbf{u}_{h}, \mathbf{v}_{h}\right)_{K}-\left(\tilde{p}_{h}, \nabla \cdot \mathbf{v}_{h}\right)_{K}+\left\langle\mathbf{v}_{h} \cdot \mathbf{n}, \tilde{p}_{h}\right\rangle_{\partial K \backslash \partial \Omega}=0 \quad \forall \mathbf{v}_{h} \in \mathbf{V}_{h}(K) .
$$

Employing the Green theorem for the two last terms of the above expression then leads to

$$
\left(\mathbf{S}^{-1}\left(\mathbf{u}_{h}+\mathbf{S} \nabla \tilde{p}_{h}\right), \mathbf{v}_{h}\right)_{K}=0 \quad \forall \mathbf{v}_{h} \in \mathbf{V}_{h}(K) \quad \forall K \in \mathcal{T}_{h},
$$

which is nothing but

$$
P_{\tilde{\mathbf{V}}_{h}}\left(-\mathbf{S} \nabla \tilde{p}_{h}\right)=\mathbf{u}_{h}
$$

The finite-dimensional spaces $M_{h}$ for the individual families and types of elements are detailed in [8] (cf. also [9, 25]); principally, they consist of piecewise polynomial spaces augmented with bubble functions. They are usually nonconforming in the sense that $M_{h} \not \subset H_{0}^{1}(\Omega)$. We also remark that whereas for a given space $M_{h}$, $\tilde{p}_{h} \in M_{h}$ satisfying (4.4a)-4.4b) is prescribed uniquely, the space $M_{h}$ itself for a given method is not defined in a unique way; in particular, there exist several different spaces for the lowest-order Raviart-Thomas elements on triangles.

For the analysis of this paper, along with (4.4a) $4.4 \mathrm{~b}$, we will only need the three following characterizing properties of the spaces $M_{h}$ :

$$
\begin{aligned}
M_{h} & \subset W_{h}\left(\mathcal{T}_{h}\right), \\
\inf _{s_{h} \in M_{h}}\left|\left\|s-s_{h}\right\|\right| & \leq C h^{k+1} \quad \forall s \in H^{k+2}(\Omega) \cap H_{0}^{1}(\Omega),
\end{aligned}
$$

$$
\left(\nabla \xi_{h}, \mathbf{v}_{h}\right)_{K}=0 \quad \forall \mathbf{v}_{h} \in \mathbf{V}_{h}(K) \Rightarrow \nabla \xi_{h}=0 \quad \forall \xi_{h} \in M_{h}(K), \forall K \in \mathcal{T}_{h} .
$$

The first property simply ensures that there is "enough continuity" in $M_{h}$, the second one guarantees that $M_{h}$ is "large enough", and the last one ensures the "compatibility" of $\nabla M_{h}$ with $\mathbf{V}_{h}$. Note that (4.6c) in particular implies $\operatorname{dim}\left(M_{h}(K)\right) \leq$ $\operatorname{dim}\left(\mathbf{V}_{h}(K)\right)+1$. Conditions (4.6a) and (4.6c) for the spaces $M_{h}$ from 8 ] are satisfied for all the elements from Table 1. Some of the spaces $M_{h}$ from [8] satisfy $H_{0}^{1}(\Omega) \cap \mathbb{P}_{k+1}\left(\mathcal{T}_{h}\right) \subset M_{h}$, whence (4.6b) easily follows. 


\section{A PRIORI ERROR ANALYSIS}

We show in this section that with the abstract result of Theorem 3.1 it is immediate to get the a priori error estimates for the flux in the form $\left\|\mid \mathbf{u}-\mathbf{u}_{h}\right\|_{*} \leq$ $\left\|\mid \mathbf{u}-I_{\mathbf{V}_{h}}(\mathbf{u})\right\|_{*}$, where $I_{\mathbf{V}_{h}}$ is the vector interpolation operator of each mixed finite element method. Consequently, we easily recover the known a priori error estimates for the flux. Then, using the postprocessing of Sections 4.4.1 and 4.4.2, we establish analogous results for the potential; here some of the estimates seem to be new. Finally, we show that the uniform discrete inf-sup condition easily follows by the postprocessing of Section 4.4 and the discrete Friedrichs inequality.

Throughout this section, we shall suppose that $\mathcal{T}_{h}$ is shape-regular with a constant $\kappa_{\mathcal{T}}$. We always give a detailed form of the estimates up to the form with the error between the exact solution and its interpolate. Obtaining the final error estimates is then a question of application of interpolation estimates, presented, e.g., in [20, 52, 56]. For the sake of completeness, we include these final results, supposing the full necessary regularity; here $C$ denotes a generic constant independent of $h$.

5.1. Estimates for the flux. A straightforward application of Theorem 3.1 gives the following result:

Theorem 5.1 (Abstract a priori estimate for the flux). Let $\mathbf{u}$ given by (1.4a)(1.4b) belong to the space $\tilde{\mathbf{H}}(\operatorname{div}, \Omega)$ and let $\mathbf{u}_{h}$ be given by (1.5a)-1.5b). Next, let $I_{\mathbf{V}_{h}}$ be the mixed interpolation operator, satisfying the commuting diagram property, see [20, Section III.3]. Then

$$
\left\|\mathbf{u}-\mathbf{u}_{h}\right\|_{*} \leq\left\|\mathbf{u}-I_{\mathbf{V}_{h}}(\mathbf{u})\right\|_{*} .
$$

Proof. Put $\mathbf{v}=\mathbf{u}_{h}, \mathbf{w}=\mathbf{u}$, and $\mathbf{t}=I_{\mathbf{V}_{h}}(\mathbf{u})$ in Theorem 3.1. This gives

$$
\left\|\mathbf{u}_{h}-\mathbf{u}\right\|\left\|_{*} \leq\left|\left\|\mathbf{u}-I_{\mathbf{V}_{h}}(\mathbf{u})\left|\|_{*}+\right| \mathcal{A}\left(\mathbf{u}_{h}-\mathbf{u}, \frac{\mathbf{u}_{h}-I_{\mathbf{V}_{h}}(\mathbf{u})}{\left\|\mathbf{u}_{h}-I_{\mathbf{V}_{h}}(\mathbf{u}) \mid\right\|_{*}}\right) \mid .\right.\right.\right.
$$

Notice that the properties of the interpolation operator $I_{\mathbf{V}_{h}}$ imply

$$
\mathcal{A}\left(\mathbf{u}_{h}-\mathbf{u}, \mathbf{u}_{h}-I_{\mathbf{V}_{h}}(\mathbf{u})\right)=0 .
$$

Indeed, it follows by subtracting (1.4a) from (1.5a) and using (2.3) that

$$
\mathcal{A}\left(\mathbf{u}_{h}-\mathbf{u}, \mathbf{v}_{h}\right)=\left(p_{h}-p, \nabla \cdot \mathbf{v}_{h}\right)
$$

for all $\mathbf{v}_{h} \in \mathbf{V}_{h}$. It suffices to put $\mathbf{v}_{h}=\mathbf{u}_{h}-I_{\mathbf{V}_{h}}(\mathbf{u})$ and to notice that $\nabla$. $\left(\mathbf{u}_{h}-I_{\mathbf{V}_{h}}(\mathbf{u})\right)=0$, which follows from (1.5b) and from the commuting diagram property [20, Proposition III.3.7], to see (5.2). Hence the result follows.

Noting that $\nabla \cdot \mathbf{u}_{h}=P_{\Phi_{h}}(f)$ by (1.5b) and using the interpolation estimates, see, e.g., [20, 52, 56] we infer from the previous results the following corollary:

Corollary $\mathbf{5 . 2}$ (A priori estimates for the flux). Let $\mathbf{u}$ be given by (1.4a)-(1.4b) and $\mathbf{u}_{h}$ by (1.5a)-(1.5b). Then

$$
\begin{aligned}
\left\|\left|\mathbf{u}-\mathbf{u}_{h}\right|\right\|_{*} & \leq C h^{k+1}, \\
\left\|\mid \mathbf{u}-\mathbf{u}_{h}\right\| \|_{*, \operatorname{div}} & \leq C h^{l+1} .
\end{aligned}
$$


5.2. Estimates for the postprocessed potential in the lowest-order Raviart-Thomas-Nédélec case. As the proof of the following theorem shows, a priori error estimates for the postprocessed potential $\tilde{p}_{h}$ under Assumption (A) are straightforward.

Theorem 5.3 (A priori estimates for the postprocessed potential $\tilde{p}_{h}$ in the lowestorder Raviart-Thomas-Nédélec case). Let Assumption (A) hold, let $\mathbf{u}, p$ be given by (1.4a $)-(1.4 \mathrm{~b}), \mathbf{u}_{h}, p_{h}$ by (1.5a)-(1.5b), and $\tilde{p}_{h}$ by (4.2a)-(4.2b). Then

$$
\begin{aligned}
& \left\|p-\tilde{p}_{h}\right\|\|=\| \mathbf{u}-\mathbf{u}_{h} \|_{*} \leq C h, \\
& \left\|p-\tilde{p}_{h}\right\|_{1} \leq C \mid\left\|p-\tilde{p}_{h}\right\| \leq C h .
\end{aligned}
$$

Proof. For the first estimate, it is sufficient to note that (2.10) in combination with (4.2a) gives $\left\|p-\tilde{p}_{h} \mid\right\|=\|\| \mathbf{u}-\mathbf{u}_{h} \|_{*}$ and use the result of Corollary 5.2. The second estimate is then directly implied by the fact that $\tilde{p}_{h} \in W_{0}\left(\mathcal{T}_{h}\right)$ and the discrete Friedrichs inequality (2.8).

5.3. Estimates for the postprocessed potential in the general case. In the general case, one no longer has (4.2a), whence $\left\|p-\tilde{p}_{h}\right\| \mid=\left\|\mathbf{u}-\mathbf{u}_{h}\right\|_{*}$ and $\left\|\left|\tilde{p}_{h}\||=|\| \mathbf{u}_{h}\right|\right\|_{*}$ no longer holds true. As, however, the following lemma shows, there is still a strong particular connection between $\|\left|\tilde{p}_{h}\right|||$ and $\left\|\left|\mathbf{u}_{h}\right|\right\|_{*}$.

Lemma 5.4 (Equivalence between the energy seminorms on $M_{h}(K)$ and $P_{\tilde{\mathbf{V}}_{h}}(-\mathbf{S}$ $\left.\left.\nabla M_{h}(K)\right)\right)$. There holds

$$
\begin{array}{r}
\left\|P _ { \tilde { \mathbf { V } } _ { h } } ( - \mathbf { S } \nabla \xi _ { h } ) | \| _ { * , K } \leq \| | \left|\xi_{h}\left\|\left.\right|_{K} \leq C_{\text {eq }} \mid\right\| P_{\tilde{\mathbf{V}}_{h}}\left(-\mathbf{S} \nabla \xi_{h}\right)\|\|_{*, K}\right.\right. \\
\forall K \in \mathcal{T}_{h}, \forall \xi_{h} \in M_{h}(K)
\end{array}
$$

and thus, in particular,

$$
||\left|\mathbf{u}_{h}\right|\left\|_{*} \leq||\left|\tilde{p}_{h}\right||| \leq C_{\mathrm{eq}}||\left|\mathbf{u}_{h}\right|\right\|_{*}
$$

More generally,

$$
\left\|\nabla \xi_{h}\right\|_{K} \leq C_{K} \sup _{\mathbf{v}_{h} \in \mathbf{V}_{h}(K)} \frac{\left(\nabla \xi_{h}, \mathbf{v}_{h}\right)_{K}}{\left\|\mathbf{v}_{h}\right\|_{K}} \quad \forall K \in \mathcal{T}_{h}, \forall \xi_{h} \in M_{h}(K) .
$$

Proof. We have

$$
\left.||\left|P_{\tilde{\mathbf{V}}_{h}}\left(-\mathbf{S} \nabla \xi_{h}\right)\right|\right|_{*, K} \leq\left\|||-\mathbf{S} \nabla \xi_{h}\left|\left\|_{*, K}=\left|\left\|\xi_{h} \mid\right\|_{K}\right.\right.\right.\right.
$$

by the fact that $P_{\tilde{\mathbf{V}}_{h}}$ is the $\mathbf{L}^{2}(K)$-orthogonal projection onto $\mathbf{V}_{h}(K)$ with respect to the scalar product $\left(\mathbf{S}^{-1} \cdot, \cdot\right)_{K}$, whose norm is $\|\cdot \cdot\| \|_{*, K}$, and by (2.10). Supposing for the moment the validity of (5.4), we now prove that the other inequality in (5.3) holds true. Let $K \in \mathcal{T}_{h}$ and $\xi_{h} \in M_{h}(K)$ be given. First note that by (5.4), the definition (4.3b) of $P_{\tilde{\mathbf{V}}_{h}}$, the Cauchy-Schwarz inequality, the assumption on $\mathbf{S}$, and (2.9), we get

$$
\begin{aligned}
\left\|\nabla \xi_{h}\right\|_{K} & \leq C_{K} \sup _{\mathbf{v}_{h} \in \mathbf{V}_{h}(K)} \frac{\left(\mathbf{S}^{-1} \mathbf{S} \nabla \xi_{h}, \mathbf{v}_{h}\right)_{K}}{\left\|\mathbf{v}_{h}\right\|_{K}}=C_{K} \sup _{\mathbf{v}_{h} \in \mathbf{V}_{h}(K)} \frac{\left(\mathbf{S}^{-1} P_{\tilde{\mathbf{V}}_{h}}\left(\mathbf{S} \nabla \xi_{h}\right), \mathbf{v}_{h}\right)_{K}}{\left\|\mathbf{v}_{h}\right\|_{K}} \\
& \leq C_{K}\left\|\mathbf{S}^{-1} P_{\tilde{\mathbf{V}}_{h}}\left(\mathbf{S} \nabla \xi_{h}\right)\right\|_{K} \leq \frac{C_{K}}{c_{\mathbf{S}, K}^{1 / 2}}\left\|P_{\tilde{\mathbf{V}}_{h}}\left(\mathbf{S} \nabla \xi_{h}\right)\right\|_{*, K} .
\end{aligned}
$$

Hence

$$
\left\|\xi_{h}\left|\left\|_{K} \leq C_{\mathbf{S}, K}^{1 / 2}\right\| \nabla \xi_{h}\left\|_{K} \leq C_{K} \frac{C_{\mathbf{S}, K}^{1 / 2}}{c_{\mathbf{S}, K}^{1 / 2}}\right\| P_{\tilde{\mathbf{V}}_{h}}\left(\mathbf{S} \nabla \xi_{h}\right)\right|\right\|_{*, K}
$$


by (2.7), the assumption on $\mathbf{S}$, and the previous estimate, which gives the right inequality in (5.3) with $C_{\mathrm{eq}}:=\max _{K \in \mathcal{T}_{h}}\left\{C_{K} C_{\mathbf{S}, K}^{1 / 2} / c_{\mathbf{S}, K}^{1 / 2}\right\}$. Finally, the validity of (5.4) on a reference element $\hat{K}$ with a constant only dependent on the maximal polynomial degree of $M_{h}(\hat{K})$ follows from (4.6c). Thus (5.4), with $C_{K}$ only dependent on the maximal polynomial degree of $M_{h}(K)$ and on $\kappa_{K}$ follows by the Piola transformation and scaling arguments.

Theorem 5.5 (A priori estimates for the postprocessed potential $\tilde{p}_{h}$ in the general case). Let $\mathbf{u}, p$ be given by (1.4a)-(1.4b) $, \mathbf{u}_{h}, p_{h}$ by (1.5a)-(1.5b), and $\tilde{p}_{h}$ by (4.4a)(4.4b). Then

$$
\begin{aligned}
\left\|p-\tilde{p}_{h}\right\| \mid & \leq C\left(\inf _{s_{h} \in M_{h}}\left\|p-s_{h}\right\| \mid\|+\| \mathbf{u}-\mathbf{u}_{h}\|\|_{*}+\left\|\mathbf{u}-P_{\tilde{\mathbf{V}}_{h}}(\mathbf{u})\right\|_{*}\right) \\
& \leq C h^{k+1} \\
\left\|p-\tilde{p}_{h}\right\|_{1} & \leq C \mid\left\|p-\tilde{p}_{h}\right\| \leq C h^{k+1} .
\end{aligned}
$$

Proof. Let $s_{h} \in M_{h}$ be arbitrary. Using (5.3), (4.5), adding and subtracting $\mathbf{u}$ and $P_{\tilde{\mathbf{V}}_{h}}(\mathbf{u})$, using that $\mathbf{u}=-\mathbf{S} \nabla p$, and finally employing the triangle inequality, the fact that $P_{\tilde{\mathbf{V}}_{h}}$ is the $\mathbf{L}^{2}(\Omega)$-orthogonal projection onto $\tilde{\mathbf{V}}_{h}$ with respect to the scalar product $\left(\mathbf{S}^{-1} \cdot, \cdot\right)$, and (2.10), we have

$$
\begin{aligned}
\left\|\tilde{p}_{h}-s_{h} \mid\right\| & \leq C_{\mathrm{eq}}\left\|\left|P_{\tilde{\mathbf{V}}_{h}}\left(\mathbf{S} \nabla\left(\tilde{p}_{h}-s_{h}\right)\right)\left\|_{*}=C_{\mathrm{eq}} \mid\right\|-\mathbf{u}_{h}-P_{\tilde{\mathbf{V}}_{h}}\left(\mathbf{S} \nabla s_{h}\right)\|\|_{*}\right.\right. \\
& =C_{\mathrm{eq}}\left\|\left|-\mathbf{u}_{h}+\mathbf{u}-\mathbf{u}+P_{\tilde{\mathbf{V}}_{h}}(\mathbf{u})+P_{\tilde{\mathbf{V}}_{h}}\left(\mathbf{S} \nabla\left(p-s_{h}\right)\right)\right|\right\|_{*} \\
& \leq C_{\mathrm{eq}}\left(\left\|\mathbf{u}-\mathbf{u}_{h}\right\|\left\|_{*}+\right\| \mathbf{u}-P_{\tilde{\mathbf{V}}_{h}}(\mathbf{u})\left\|_{*}+\right\| p-s_{h} \| \mid\right) .
\end{aligned}
$$

Thus (5.5) follows by the triangle inequality $\left\|\left|p-\tilde{p}_{h}\left\|\left|\leq\left\||| p-s_{h}\right\|\right|+\right\| \tilde{p}_{h}-s_{h}\right|\right\|$, (4.6b), Corollary [5.2, and the approximation properties of $P_{\tilde{\mathbf{V}}_{h}}$. Estimate (5.6) then again follows immediately by the discrete Friedrichs inequality (2.8).

5.4. Estimates for the original potential. In this section, we easily recover the estimates for the original potential $p_{h}$ from the previous results.

Theorem 5.6 (A priori estimates for the original potential $p_{h}$ ). Let $\mathbf{u}, p$ be given by (1.4a) -(1.4b), $\mathbf{u}_{h}, p_{h}$ by (1.5a)-(1.5b), and $\tilde{p}_{h}$ by (4.2a) -(4.2b) or (4.4a ) (4.4b) . Then

$$
\left\|p-p_{h}\right\| \leq\left\|p-P_{\Phi_{h}}(p)\right\|+\left\|p-\tilde{p}_{h}\right\| \leq C h^{l+1} .
$$

Proof. Using (4.4a), adding and subtracting $P_{\Phi_{h}}(p)$, employing the triangle inequality, and finally the fact that $P_{\Phi_{h}}$ is the $L^{2}(\Omega)$-orthogonal projection onto $\Phi_{h}$, we have

$$
\begin{aligned}
\left\|p-p_{h}\right\| & =\left\|p-P_{\Phi_{h}}\left(\tilde{p}_{h}\right)\right\|=\left\|p-P_{\Phi_{h}}(p)+P_{\Phi_{h}}\left(p-\tilde{p}_{h}\right)\right\| \\
& \leq\left\|p-P_{\Phi_{h}}(p)\right\|+\left\|P_{\Phi_{h}}\left(p-\tilde{p}_{h}\right)\right\| \leq\left\|p-P_{\Phi_{h}}(p)\right\|+\left\|p-\tilde{p}_{h}\right\| .
\end{aligned}
$$

The final estimate then follows by Theorem 5.5 and the approximation properties of $P_{\Phi_{h}}$.

5.5. Superconvergence estimates for the original potential. For the sake of completeness, we show in this section the superconvergence estimates for the original potential $p_{h}$, following essentially [30, 20, Section V.3], and [29]. Let $\mathbf{e}_{i} \in \mathbb{R}^{d}$ be such that $\mathbf{e}_{i}^{i}=1$ and $\mathbf{e}_{i}^{j}=0$ for $i \neq j$. 
Assumption (B) (Elliptic regularity). For each $g_{h} \in \Phi_{h}$, the weak solution of the problem

$$
\begin{aligned}
\mathbf{r} & =-\mathbf{S} \nabla q & & \text { in } \Omega, \\
\nabla \cdot \mathbf{r} & =g_{h} & & \text { in } \Omega, \\
q & =0 & & \text { on } \partial \Omega
\end{aligned}
$$

satisfies

$$
\|q\|_{2}+|\mathbf{r}|_{1} \leq C_{\mathrm{ER}}\left\|g_{h}\right\|
$$

Theorem 5.7 (Superconvergence estimates for the original potential $p_{h}$ ). Let $\mathbf{u}$, $p$ be given by (1.4a) -(1.4b) and $\mathbf{u}_{h}, p_{h}$ by (1.5a - (1.5b). Next, let Assumption (B) hold. Then if $l=k$,

$$
\left\|P_{\Phi_{h}}(p)-p_{h}\right\| \leq C h\left(\left\|\mathbf{u}-I_{\mathbf{V}_{h}}(\mathbf{u})\right\|_{*}+\left\|\nabla \cdot\left(\mathbf{u}-I_{\mathbf{V}_{h}}(\mathbf{u})\right)\right\|\right) \leq C h^{k+2},
$$

and if $k \geq 1$ and $\left(\mathbf{u}-I_{\mathbf{V}_{h}} \mathbf{u}, \mathbf{e}_{i}\right)_{K}=0$ for each $i=1, \ldots, d$ and $K \in \mathcal{T}_{h}$,

$$
\left\|P_{\Phi_{h}}(p)-p_{h}\right\| \leq C h\left(\left\|\mathbf{u}-I_{\mathbf{V}_{h}}(\mathbf{u})\right\|_{*}\right) \leq C h^{k+2} .
$$

Proof. We use the characterization

$$
\left\|P_{\Phi_{h}}(p)-p_{h}\right\|=\sup _{g_{h} \in \Phi_{h}} \frac{\left(P_{\Phi_{h}}(p)-p_{h}, g_{h}\right)}{\left\|g_{h}\right\|} .
$$

Next, using the definition (4.3a) of the orthogonal projection $P_{\Phi_{h}}$, the fact that $\nabla \cdot I_{\mathbf{V}_{h}}(\mathbf{r})=g_{h}$, and subtracting (1.5a) from (1.4a), we get

$$
\begin{aligned}
\left(P_{\Phi_{h}}(p)-p_{h}, g_{h}\right)= & \left(p-p_{h}, g_{h}\right)=\left(p-p_{h}, \nabla \cdot I_{\mathbf{V}_{h}}(\mathbf{r})\right)=\left(\mathbf{S}^{-1}\left(\mathbf{u}-\mathbf{u}_{h}\right), I_{\mathbf{V}_{h}}(\mathbf{r})\right) \\
= & \left(\mathbf{S}^{-1}\left(\mathbf{u}-I_{\mathbf{V}_{h}}(\mathbf{u})\right), I_{\mathbf{V}_{h}}(\mathbf{r})\right)+\left(\mathbf{S}^{-1}\left(I_{\mathbf{V}_{h}}(\mathbf{u})-\mathbf{u}_{h}\right), I_{\mathbf{V}_{h}}(\mathbf{r})\right) \\
= & \left(\mathbf{S}^{-1}\left(\mathbf{u}-I_{\mathbf{V}_{h}}(\mathbf{u})\right), I_{\mathbf{V}_{h}}(\mathbf{r})-\mathbf{r}\right)+\left(\mathbf{S}^{-1}\left(\mathbf{u}-I_{\mathbf{V}_{h}}(\mathbf{u})\right), \mathbf{r}\right) \\
& +\left(\mathbf{S}^{-1}\left(I_{\mathbf{V}_{h}}(\mathbf{u})-\mathbf{u}_{h}\right), I_{\mathbf{V}_{h}}(\mathbf{r})-\mathbf{r}\right)+\left(\mathbf{S}^{-1}\left(I_{\mathbf{V}_{h}}(\mathbf{u})-\mathbf{u}_{h}\right), \mathbf{r}\right) .
\end{aligned}
$$

We now first note that for the last term, we have

$$
\left(\mathbf{S}^{-1}\left(I_{\mathbf{V}_{h}}(\mathbf{u})-\mathbf{u}_{h}\right), \mathbf{r}\right)=-\left(I_{\mathbf{V}_{h}}(\mathbf{u})-\mathbf{u}_{h}, \nabla q\right)=\left(\nabla \cdot\left(I_{\mathbf{V}_{h}}(\mathbf{u})-\mathbf{u}_{h}\right), q\right)=0,
$$

employing (5.7a), the Green theorem, and the fact that $\nabla \cdot\left(I_{\mathbf{V}_{h}}(\mathbf{u})-\mathbf{u}_{h}\right)=0$. Next, the first term can be estimated by employing (5.8),

$$
\begin{aligned}
\left(\mathbf{S}^{-1}\left(\mathbf{u}-I_{\mathbf{V}_{h}}(\mathbf{u})\right), I_{\mathbf{V}_{h}}(\mathbf{r})-\mathbf{r}\right) & \leq\left.\left\|\left|\mathbf{u}-I_{\mathbf{V}_{h}}(\mathbf{u})\right|\right\|\right|_{*}\left\|I_{\mathbf{V}_{h}}(\mathbf{r})-\mathbf{r}\right\| \|_{*} \\
& \leq C h\left\|\mathbf{u}-I_{\mathbf{V}_{h}}(\mathbf{u})\right\| \|_{*}|\mathbf{r}|_{1} \\
& \leq C C_{\mathrm{ER}} h\left|\left\|\mathbf{u}-I_{\mathbf{V}_{h}}(\mathbf{u})\right\|\right|_{*}\left\|g_{h}\right\| .
\end{aligned}
$$

The third term can be estimated similarly, using in addition the triangle inequality and (5.1). Finally, there are two ways to estimate the second term. First,

$$
\begin{aligned}
\left(\mathbf{S}^{-1}\left(\mathbf{u}-I_{\mathbf{V}_{h}}(\mathbf{u})\right), \mathbf{r}\right) & =-\left(\mathbf{u}-I_{\mathbf{V}_{h}}(\mathbf{u}), \nabla q\right)=\left(\nabla \cdot\left(\mathbf{u}-I_{\mathbf{V}_{h}}(\mathbf{u})\right), q\right) \\
& =\left(\nabla \cdot\left(\mathbf{u}-I_{\mathbf{V}_{h}}(\mathbf{u})\right), q-\pi_{0}(q)\right) \\
& \leq C_{\mathrm{P}}^{\frac{1}{2}} h\left\|\nabla \cdot\left(\mathbf{u}-I_{\mathbf{V}_{h}}(\mathbf{u})\right)\right\||q|_{1} \\
& \leq C_{\mathrm{P}}^{\frac{1}{2}} C_{\mathrm{ER}} h\left\|\nabla \cdot\left(\mathbf{u}-I_{\mathbf{V}_{h}}(\mathbf{u})\right)\right\|\left\|g_{h}\right\|
\end{aligned}
$$


employing (5.7a), the Green theorem, the fact that $\left(\nabla \cdot\left(\mathbf{u}-I_{\mathbf{V}_{h}}(\mathbf{u})\right), 1\right)_{K}=0$ for all $K \in \mathcal{T}_{h}$, the Poincaré inequality (2.13), and (5.8). Alternatively, if $k \geq 1$ and $\left(\mathbf{u}-I_{\mathbf{V}_{h}} \mathbf{u}, \mathbf{e}_{i}\right)_{K}=0$ for each $i=1, \ldots, d$ and $K \in \mathcal{T}_{h}$, then

$$
\begin{aligned}
\left(\mathbf{S}^{-1}\left(\mathbf{u}-I_{\mathbf{V}_{h}}(\mathbf{u})\right), \mathbf{r}\right) & =\left(I_{\mathbf{V}_{h}}(\mathbf{u})-\mathbf{u}, \nabla q\right)=\left(I_{\mathbf{V}_{h}}(\mathbf{u})-\mathbf{u}, \nabla q-\pi_{0}(\nabla q)\right) \\
& \leq C_{\mathrm{P}}^{\frac{1}{2}} h\left\|I_{\mathbf{V}_{h}}(\mathbf{u})-\mathbf{u}\right\||q|_{2} \leq C_{\mathrm{P}}^{\frac{1}{2}} C_{\mathrm{ER}} C_{\mathbf{S}, \Omega}^{\frac{1}{2}} h\left\|\mathbf{u}-I_{\mathbf{V}_{h}}(\mathbf{u})\right\|\left\|_{*}\right\| g_{h} \|,
\end{aligned}
$$

employing also the Poincaré inequality (2.13), the assumption on $\mathbf{S}$, and the definition of the energy norm (2.9). Combining the above estimates proves the assertions of the theorem.

5.6. Superconvergence estimates for the postprocessed potential. Using the results of the previous section, we establish here in a straightforward way superconvergence estimates for the postprocessed potential $\tilde{p}_{h}$.

Theorem 5.8 (Superconvergence estimates for the postprocessed potential $\tilde{p}_{h}$ ). Let $\mathbf{u}, p$ be given by (1.4a) (1.4b), $\mathbf{u}_{h}, p_{h}$ by (1.5a) (1.5b), and $\tilde{p}_{h}$ by (4.2a - 4.2b) or (4.4a)-(4.4b). Then

$$
\left\|p-\tilde{p}_{h}\right\| \leq C h\left\|p-\tilde{p}_{h}\right\| \mid+\left\|P_{\Phi_{h}}(p)-p_{h}\right\| .
$$

If, in particular, Assumption (B) holds and if either $l=k$ or $k \geq 1$ and ( $\mathbf{u}-$ $\left.I_{\mathbf{V}_{h}} \mathbf{u}, \mathbf{e}_{i}\right)_{K}=0$ for each $i=1, \ldots, d$ and $K \in \mathcal{T}_{h}$, then

$$
\left\|p-\tilde{p}_{h}\right\| \leq C h^{k+2} \text {. }
$$

Proof. We have, using the triangle inequality, the fact that $P_{\Phi_{h}}$ is the $L^{2}(\Omega)$ orthogonal projection onto $\Phi_{h}$, 4.4a), and the Poincaré inequality (2.13),

$$
\begin{aligned}
\left\|p-\tilde{p}_{h}\right\| & =\left\|p-\tilde{p}_{h}-P_{\Phi_{h}}\left(p-\tilde{p}_{h}\right)+P_{\Phi_{h}}\left(p-\tilde{p}_{h}\right)\right\| \\
& \leq\left\|p-\tilde{p}_{h}-\pi_{0}\left(p-\tilde{p}_{h}\right)\right\|+\left\|P_{\Phi_{h}}(p)-p_{h}\right\| \\
& \leq C_{\mathrm{P}}^{\frac{1}{2}} h\left|p-\tilde{p}_{h}\right|_{1}+\left\|P_{\Phi_{h}}(p)-p_{h}\right\| \\
& \leq \frac{C_{\mathrm{P}}^{1 / 2} h}{c_{\mathbf{S}, \Omega}^{1 / 2}}\left\|p-\tilde{p}_{h}\right\|+\left\|P_{\Phi_{h}}(p)-p_{h}\right\|
\end{aligned}
$$

5.7. Uniform discrete inf-sup condition. As a complement, we show here that the postprocessing of Section 4.4, Lemma 5.4, and the discrete Friedrichs inequality (2.8) imply:

Theorem 5.9 (Uniform discrete inf-sup condition). There holds

$$
\inf _{\phi_{h} \in \Phi_{h}} \sup _{\mathbf{v}_{h} \in \mathbf{V}_{h}} \frac{\left(\phi_{h}, \nabla \cdot \mathbf{v}_{h}\right)}{\left\|\phi_{h}\right\|\left\|\mathbf{v}_{h}\right\|} \geq \frac{1}{C_{\mathrm{DF}}^{\frac{1}{2}} C_{\mathrm{eq}}}
$$

where $C_{\mathrm{DF}}$ is the constant from (2.8) and $C_{\mathrm{eq}}$ is the constant from (5.3) for $\mathbf{S}=\mathbb{I}$.

Proof. We have to show that for all $\phi_{h} \in \Phi_{h}$, there exists $\mathbf{v}_{h} \in \mathbf{V}_{h}$ such that $\left(\phi_{h}, \nabla \cdot \mathbf{v}_{h}\right) \geq\left\|\phi_{h}\right\|\left\|\mathbf{v}_{h}\right\| / C_{\mathrm{DF}}^{\frac{1}{2}} / C_{\mathrm{eq}}$. Consider $\mathbf{v}_{h} \in \mathbf{V}_{h}$ and $q_{h} \in \Phi_{h}$ the solution to

$$
\begin{aligned}
\left(\mathbf{v}_{h}, \mathbf{w}_{h}\right)-\left(q_{h}, \nabla \cdot \mathbf{w}_{h}\right) & =0 & & \forall \mathbf{w}_{h} \in \mathbf{V}_{h} \\
\left(\nabla \cdot \mathbf{v}_{h}, \psi_{h}\right) & =\left(\phi_{h}, \psi_{h}\right) & & \forall \psi_{h} \in \Phi_{h}
\end{aligned}
$$


Let $\tilde{q}_{h}$ be the postprocessing of $\mathbf{v}_{h}, q_{h}$ of Section 4.4.1 or 4.4.2 (with $\mathbf{S}=\mathbb{I}$ ). Then

$$
\begin{aligned}
\left\|\mathbf{v}_{h}\right\|^{2} & =\left(\mathbf{v}_{h}, \mathbf{v}_{h}\right)=\left(q_{h}, \nabla \cdot \mathbf{v}_{h}\right)=\left(\tilde{q}_{h}, \nabla \cdot \mathbf{v}_{h}\right)=\left(\tilde{q}_{h}, \phi_{h}\right) \\
& \leq\left\|\tilde{q}_{h}\right\|\left\|\phi_{h}\right\| \leq C_{\mathrm{DF}}^{\frac{1}{2}}\left\|\nabla \tilde{q}_{h}\right\|\left\|\phi_{h}\right\| \leq C_{\mathrm{DF}}^{\frac{1}{2}} C_{\mathrm{eq}}\left\|\mathbf{v}_{h}\right\|\left\|\phi_{h}\right\|
\end{aligned}
$$

by (5.10a) with $\mathbf{w}_{h}=\mathbf{v}_{h}$, the properties of the postprocessing, (5.10b) which gives $\nabla \cdot \mathbf{v}_{h}=\phi_{h}$, the Cauchy-Schwarz inequality, (2.8), and (5.3), whence

$$
\left\|\mathbf{v}_{h}\right\| \leq C_{\mathrm{DF}}^{\frac{1}{2}} C_{\mathrm{eq}}\left\|\phi_{h}\right\| .
$$

The desired result follows by employing this last inequality in

$$
\left(\phi_{h}, \nabla \cdot \mathbf{v}_{h}\right)=\left\|\phi_{h}\right\|^{2} \geq \frac{\left\|\phi_{h}\right\|\left\|\mathbf{v}_{h}\right\|}{C_{\mathrm{DF}}^{\frac{1}{2}} C_{\mathrm{eq}}} .
$$

Note that by using the fact that $\nabla \cdot \mathbf{v}_{h}=\phi_{h}$, uniform discrete inf-sup condition with $\left\|\mathbf{v}_{h}\right\|$ replaced by $\left|\left\|\mathbf{v}_{h} \mid\right\|_{* \text {,div }}(\right.$ with $\mathbf{S}=\mathbb{I}$ ) easily follows.

\section{A POSTERIORI ERROR ANALYSIS}

We show in this section that with the abstract results of Theorems 3.1 or 3.3 it is also immediate to get an optimal framework for a posteriori error estimates for the flux in mixed finite element methods. For the potential, a similar framework developed in 62, 41, 35, is adopted. We finally give fully computable versions of all the estimates, prove their local efficiency, discuss their robustness, and present some extensions.

6.1. Estimates for the flux. We state and prove here our a posteriori error estimates for the flux, first in an abstract and then in a fully computable form.

6.1.1. Abstract estimates. An application of Theorem 3.1 gives the following result, which we state as generally as possible (without any notion of a numerical scheme); in practice, $\mathbf{u}_{h}$ is given by (1.5a) $1.5 \mathrm{~b}$.

Theorem 6.1 (Abstract a posteriori estimate for the flux and its efficiency). Let $\mathbf{u}$ be given by (1.4a - (1.4b) and let $\mathbf{u}_{h} \in \mathbf{H}(\operatorname{div}, \Omega)$ such that $\nabla \cdot \mathbf{u}_{h}=P_{\Phi_{h}}(f)$ be arbitrary. Then

$$
\left\|\mathbf{u}-\mathbf{u}_{h}\left|\left\|_{*} \leq \inf _{s \in H_{0}^{1}(\Omega)}\right\| \mathbf{u}_{h}+\mathbf{S} \nabla s\right|\right\|_{*}+\eta_{\mathrm{R}} \leq\left\|\mathbf{u}-\mathbf{u}_{h}\right\|_{*}+\eta_{\mathrm{R}},
$$

where

$$
\eta_{\mathrm{R}}:=\left\{\sum_{K \in \mathcal{T}_{h}} \frac{C_{\mathrm{P}} h_{K}^{2}}{c_{\mathrm{S}, K}}\left\|f-P_{\Phi_{h}}(f)\right\|_{K}^{2}\right\}^{\frac{1}{2}} .
$$

Proof. The right inequality in (6.1) is straightforward by putting $s=p$ and noticing that $\mathbf{u}=-\mathbf{S} \nabla p$ by the equivalence of (1.2) and (1.4a $-(1.4 \mathrm{~b})$. For the left one, put $\mathbf{v}=\mathbf{u}, \mathbf{w}=\mathbf{u}_{h}$, and $\mathbf{t}=-\mathbf{S} \nabla s$, with $s \in H_{0}^{1}(\Omega)$ arbitrary, in Theorem 3.1 This gives

$$
\left\|\mathbf{u}-\mathbf{u}_{h}\left|\left\|_{*} \leq\left|\left\|\mathbf{u}_{h}+\mathbf{S} \nabla s\right\|_{*}+\right| \mathcal{A}\left(\mathbf{u}-\mathbf{u}_{h}, \frac{\mathbf{u}+\mathbf{S} \nabla s}{\|\mathbf{u}+\mathbf{S} \nabla s\|_{*}}\right) \mid .\right.\right.\right.
$$


Next put $\varphi:=(p-s) /\|p-s\| \in \in H_{0}^{1}(\Omega)$ and rewrite the second term of the above expression as $\left|\mathcal{A}\left(\mathbf{u}-\mathbf{u}_{h},-\mathbf{S} \nabla \varphi\right)\right|$, employing $\mathbf{u}=-\mathbf{S} \nabla p$ and (2.10). Next, by the equivalent definition of the weak solution (2.5),

$$
\mathcal{A}(\mathbf{u},-\mathbf{S} \nabla \varphi)=(f, \varphi)
$$

whereas

$$
\mathcal{A}\left(\mathbf{u}_{h},-\mathbf{S} \nabla \varphi\right)=-\left(\mathbf{u}_{h}, \nabla \varphi\right)=\left(P_{\Phi_{h}}(f), \varphi\right)
$$

by (2.3), the Green theorem, and the assumption on $\mathbf{u}_{h}$. Hence

$$
\mathcal{A}\left(\mathbf{u}-\mathbf{u}_{h},-\mathbf{S} \nabla \varphi\right)=\left(f-P_{\Phi_{h}}(f), \varphi\right) .
$$

This last expression can easily be estimated by

$$
\begin{aligned}
\left(f-P_{\Phi_{h}}(f), \varphi\right) & =\sum_{K \in \mathcal{T}_{h}}\left(f-P_{\Phi_{h}}(f), \varphi\right)_{K} \\
& =\sum_{K \in \mathcal{T}_{h}}\left(f-P_{\Phi_{h}}(f), \varphi-\pi_{0}(\varphi)\right)_{K} \\
& \leq \sum_{K \in \mathcal{T}_{h}}\left\|f-P_{\Phi_{h}}(f)\right\|_{K}\left\|\varphi-\pi_{0}(\varphi)\right\|_{K} \\
& \leq \sum_{K \in \mathcal{T}_{h}}\left\|f-P_{\Phi_{h}}(f)\right\|_{K} C_{\mathrm{P}}^{\frac{1}{2}} h_{K}\|\nabla \varphi\|_{K} \\
& \leq \sum_{K \in \mathcal{T}_{h}}\left\|f-P_{\Phi_{h}}(f)\right\|_{K} \frac{C_{\mathrm{P}}^{1 / 2}}{c_{\mathbf{S}, K}^{1 / 2}} h_{K}\|\varphi\|_{K} \leq \eta_{\mathrm{R}}\|\varphi\|,
\end{aligned}
$$

employing the fact that zero-order polynomials are always in $\Phi_{h}$, which implies $\left(f-P_{\Phi_{h}}(f), \varphi\right)_{K}=\left(f-P_{\Phi_{h}}(f), \varphi-\pi_{0}(\varphi)\right)_{K}$, the Cauchy-Schwarz inequality, the Poincaré inequality (2.13), (2.7), and once again the Cauchy-Schwarz inequality. The assertion of the theorem follows by the fact that $\|\varphi\| \|=1$.

Remark 6.2 (Nature of the estimate of Theorem 6.1). Theorem 6.1 shows that the error in a vector field $\mathbf{u}_{h} \in \mathbf{H}(\operatorname{div}, \Omega)$ such that $\nabla \cdot \mathbf{u}_{h}=P_{\Phi_{h}}(f)$ is measured by how close $\mathbf{u}_{h}$ is to a flux of a $H_{0}^{1}(\Omega)$-potential plus the residual term $\eta_{\mathrm{R}}$.

Remark 6.3 (General form of the residual term). Note that the condition $\nabla \cdot \mathbf{u}_{h}=$ $P_{\Phi_{h}}(f)$ in Theorem 6.1 (and below) may easily be replaced by $\left(\nabla \cdot \mathbf{u}_{h}, 1\right)_{K}=(f, 1)_{K}$ for all $K \in \mathcal{T}_{h}$, which is completely sufficient. The residual term then changes correspondingly to $\eta_{\mathrm{R}}:=\left\{\sum_{K \in \mathcal{T}_{h}} C_{\mathrm{P}} h_{K}^{2} / c_{\mathbf{S}, K}\left\|f-\nabla \cdot \mathbf{u}_{h}\right\|_{K}^{2}\right\}^{\frac{1}{2}}$.

Remark 6.4 (Residual term in mixed finite element methods). The term $\eta_{\mathrm{R}}$ (6.2) is sometimes referred to as the "data oscillation term", because it only depends on the variation of the source function $f$, and considered separately from the actual a posteriori error estimate. If $f \in H^{l+1}\left(\mathcal{T}_{h}\right)$, this term is clearly of order $\mathcal{O}\left(h^{l+2}\right)$. Thus it is superconvergent for those mixed finite elements methods where $\left\|\mathbf{u}-\mathbf{u}_{h} \mid\right\|_{*}$ is of order $\mathcal{O}\left(h^{l+1}\right)$, namely the Raviart-Thomas-Nédélec ones. This is, however, not always the case, namely for the Brezzi-Douglas-Marini family, where $\left\|\mathbf{u}-\mathbf{u}_{h}\right\|_{*}$ is of order $\mathcal{O}\left(h^{l+2}\right)$. In the second case, in particular, it is important not to omit $\eta_{\mathrm{R}}$ from the estimate and use $h_{K}\left\|f-P_{\Phi_{h}}(f)\right\|_{K}$ with the correct weight given by the Poincaré constant $C_{\mathrm{P}}$ and the material constant $c_{\mathbf{S}, K}$. 
Remark 6.5 (Efficiency of the abstract estimate of Theorem 6.1). When the term $\eta_{\mathrm{R}}$ is superconvergent (see Remark 6.4), the estimate of Theorem 6.1 is optimal, i.e., it also represents a lower bound for the error, up to $\eta_{\mathrm{R}}$. In Theorem 6.8 below we will see that (local) efficiency also holds for $\eta_{\mathrm{R}}$ in any case. Another possibility to work with the term $\eta_{\mathrm{R}}$ is to derive estimates in the $\||\cdot|\|_{*, \mathrm{div}}$-norm, as it is done below.

Employing Theorem 3.3 instead of Theorem 3.1, we can easily get the following slightly improved version of Theorem 6.1

Corollary 6.6 (Improved abstract a posteriori estimate for the flux and its efficiency). Let $\mathbf{u}$ be given by (1.4a) -(1.4b) and let $\mathbf{u}_{h} \in \mathbf{H}(\operatorname{div}, \Omega)$ such that $\nabla \cdot \mathbf{u}_{h}=$ $P_{\Phi_{h}}(f)$ is arbitrary. Then

$$
\left\|\left|\mathbf{u}-\mathbf{u}_{h}\right|\right\|_{*}^{2} \leq \inf _{s \in H_{0}^{1}(\Omega)}\left\|\mathbf{u}_{h}+\mathbf{S} \nabla s \mid\right\|_{*}^{2}+\eta_{\mathrm{R}}^{2} \leq\left\|\mathbf{u}-\mathbf{u}_{h}\right\|_{*}^{2}+\eta_{\mathrm{R}}^{2} .
$$

This version is particularly suitable to derive in a straightforward way an estimate in the $\||\cdot|\|_{*, \operatorname{div}}$-norm:

Theorem 6.7 (Abstract $\||\cdot|\|_{*, \text { div }}$-norm a posteriori estimate for the flux and its efficiency). Let $\mathbf{u}$ be given by (1.4a) -1.4b) and let $\mathbf{u}_{h} \in \mathbf{H}(\operatorname{div}, \Omega)$ such that $\nabla \cdot \mathbf{u}_{h}=P_{\Phi_{h}}(f)$ is arbitrary. Then

$\left\|\left|\mathbf{u}-\mathbf{u}_{h}\right|\right\|_{*, \operatorname{div}}^{2} \leq \inf _{s \in H_{0}^{1}(\Omega)}\left\|\mathbf{u}_{h}+\mathbf{S} \nabla s\right\|_{*}^{2}+\left\|f-P_{\Phi_{h}}(f)\right\|^{2}+\eta_{\mathrm{R}}^{2} \leq\left\|\mathbf{u}-\mathbf{u}_{h}\right\|_{*, \operatorname{div}}^{2}+\eta_{\mathrm{R}}^{2}$.

Note that now the term $\eta_{\mathrm{R}}$, by its definition, converges by one order faster than $\left\|f-P_{\Phi_{h}}(f)\right\|$. Hence, in contrast to Theorem 6.1 (see also Remark 6.5), the $\|\cdot \cdot\| \|_{*, \text { div }^{-}}$ norm setting gives an optimal global abstract efficiency, up to the term $\eta_{\mathrm{R}}$, which is now always superconvergent (also in the Brezzi-Douglas-Marini-like cases). On the other hand, however, the term $\left\|f-P_{\Phi_{h}}(f)\right\|$ is generally of order $\mathcal{O}\left(h^{l+1}\right)$, which may dominate the error in the Brezzi-Douglas-Marini-like cases, where $\left\|\mathbf{u}-\mathbf{u}_{h} \mid\right\|_{*}$ is of order $\mathcal{O}\left(h^{l+2}\right)$. As this term is entirely data dependent, we believe that, although Theorem 6.7 gives an optimal abstract estimate and efficiency, the $\||\cdot|\|_{* \text {,div }}$-norm estimate is not suitable for a posteriori error estimation, as previously noted in, e.g., [4, Remark 3.4].

6.1.2. Fully computable estimates. Employing Corollary 6.6 and Theorem 6.7, we see that in order to give a fully computable a posteriori error estimate, we only need to specify a function $s \in H_{0}^{1}(\Omega)$. This choice is of course particularly important for the precision of the estimate and it is also crucial in order to prove the local efficiency. Clearly, $-\mathbf{S} \nabla s$ has to be as close as possible to $\mathbf{u}_{h}$. In view of this fact, we are led to first consider $\tilde{p}_{h}$ given by (4.2a) -4.2b) in the lowest-order Raviart-Thomas-Nédélec case and by (4.4a - 4.4b) otherwise, for $\mathbf{u}_{h}$ the mixed finite element solution given by (1.5a)-(1.5b). Recall that $\mathbf{u}_{h}$ directly equals $-\mathbf{S} \nabla \tilde{p}_{h}$ under Assumption (A) and that $\mathbf{u}_{h}$ is very close to $-\mathbf{S} \nabla \tilde{p}_{h}$ in general by (4.5). The last step is then to "smooth" $\tilde{p}_{h}$ into a conforming function and for exactly this reason, in Section 2.3 we have introduced the averaging operator. Hence (a general version of) our fully computable a posteriori error estimate is as follows:

Theorem 6.8 (Fully computable a posteriori estimates for the flux). Let $\mathbf{u}$ be given by (1.4a) - (1.4b) and let $\mathbf{u}_{h} \in \mathbf{H}(\operatorname{div}, \Omega)$ such that $\nabla \cdot \mathbf{u}_{h}=P_{\Phi_{h}}(f)$ and $\tilde{p}_{h} \in \mathbb{R}_{n}\left(\mathcal{T}_{h}\right)$ for some $n \geq 1$ be arbitrary. Let the potential estimator be given by

$$
\eta_{\mathrm{P}, K}:=\left\|\mathbf{u}_{h}+\mathbf{S} \nabla\left(\mathcal{I}_{\mathrm{av}}\left(\tilde{p}_{h}\right)\right)\right\|_{*, K},
$$


the residual estimator by

$$
\eta_{\mathrm{R}, K}:=\frac{C_{\mathrm{P}}^{1 / 2} h_{K}}{c_{\mathbf{S}, K}^{1 / 2}}\left\|f-P_{\Phi_{h}}(f)\right\|_{K},
$$

and the divergence estimator by

$$
\eta_{\mathrm{D}, K}:=\left\|f-P_{\Phi_{h}}(f)\right\|_{K} .
$$

Then

$$
\begin{aligned}
\left\|\left|\mathbf{u}-\mathbf{u}_{h}\right|\right\|_{*}^{2} & \leq \sum_{K \in \mathcal{T}_{h}}\left(\eta_{\mathrm{P}, K}^{2}+\eta_{\mathrm{R}, K}^{2}\right), \\
\left\|\left|\mathbf{u}-\mathbf{u}_{h}\right|\right\|_{*, \text { div }}^{2} & \leq \sum_{K \in \mathcal{T}_{h}}\left(\eta_{\mathrm{P}, K}^{2}+\eta_{\mathrm{R}, K}^{2}+\eta_{\mathrm{D}, K}^{2}\right) .
\end{aligned}
$$

Remark 6.9 (Constants in Theorem 6.8). Note that there are no undetermined constants in the estimates of Theorem 6.8. Moreover, the leading estimators $\eta_{\mathrm{P}, K}$ and $\eta_{\mathrm{D}, K}$ are completely constant-free and the only constant (recall from (2.13) that $C_{\mathrm{P}}=1 / \pi^{2}$ ) appears in the residual estimator $\eta_{\mathrm{R}, K}$, which is likely to be superconvergent; see Remark 6.4.

6.2. Estimates for the potential. We state and prove here our a posteriori error estimates for the potential, first in an abstract way and then in a fully computable form.

6.2.1. Abstract estimates. Building on the approaches of [62, Lemma 7.1] and 41, Lemma 4.4], the following can be shown; cf. [35, Lemma 4.1]:

Theorem 6.10 (Abstract a posteriori estimate for the potential and its efficiency). Let $p$ be the weak potential given by (1.2) and let $\tilde{p}_{h} \in H^{1}\left(\mathcal{T}_{h}\right)$ is arbitrary. Then

$$
\begin{aligned}
\left.\left\|p-\tilde{p}_{h}\right\|\right|^{2} \leq & \inf _{s \in H_{0}^{1}(\Omega)}\left\|\tilde{p}_{h}-s\right\|^{2} \\
& +\inf _{\mathbf{t} \in \mathbf{H}(\operatorname{div}, \Omega)} \sup _{\varphi \in H_{0}^{1}(\Omega),\|\varphi\| \|=1}\left((f-\nabla \cdot \mathbf{t}, \varphi)-\left(\mathbf{S} \nabla \tilde{p}_{h}+\mathbf{t}, \nabla \varphi\right)\right)^{2} \\
\leq & 2\left\|p-\tilde{p}_{h}\right\|^{2} .
\end{aligned}
$$

Remark 6.11 (Nature of the estimate of Theorem 6.10). Theorem 6.10 shows that the error in a potential $\tilde{p}_{h} \in H^{1}\left(\mathcal{T}_{h}\right)$ is measured by how close $\tilde{p}_{h}$ is to the space $H_{0}^{1}(\Omega)$, how close the approximate diffusive flux $-\mathbf{S} \nabla \tilde{p}_{h}$ is to the space $\mathbf{H}(\operatorname{div}, \Omega)$, and how small the residual $f-\nabla \cdot \mathbf{t}$ can be.

6.2.2. Fully computable estimates in the energy norm. Analogously to the proof of Theorem 6.1 we have the following result. We again state it generally; in practice, it will be used for the postprocessed approximation $\tilde{p}_{h}$ of Section 4.4 and the mixed finite element approximate flux $\mathbf{u}_{h}$ given by (1.5a)-11.5b). Recall in this respect that the postprocessed potential $\tilde{p}_{h}$ belongs to $W_{0}\left(\mathcal{T}_{h}\right)$ and that $\|\cdot \mid\|$ is a norm on $W_{0}\left(\mathcal{T}_{h}\right)$ thanks to the discrete Friedrichs inequality (2.8), whence the justification of the "energy norm" (and not just seminorm) in the title of this section.

Theorem 6.12 (Fully computable energy a posteriori estimate for the potential). Let $p$ be given by (1.2) and let $\tilde{p}_{h} \in \mathbb{R}_{n}\left(\mathcal{T}_{h}\right)$ for some $n \geq 1$ and $\mathbf{u}_{h} \in \mathbf{H}(\operatorname{div}, \Omega)$ 
such that $\nabla \cdot \mathbf{u}_{h}=P_{\Phi_{h}}(f)$ be arbitrary. Let the nonconformity estimator be given by

$$
\eta_{\mathrm{NC}, K}:=\mid\left\|\tilde{p}_{h}-\mathcal{I}_{\mathrm{av}}\left(\tilde{p}_{h}\right)\right\|_{K},
$$

the diffusive flux estimator by

$$
\eta_{\mathrm{DF}, K}:=\left\|\mathbf{u}_{h}+\mathbf{S} \nabla \tilde{p}_{h}\right\|_{*, K},
$$

and the residual estimator by (6.4). Then

$$
\left.\left\|p-\tilde{p}_{h}\right\|\right|^{2} \leq \sum_{K \in \mathcal{T}_{h}}\left\{\eta_{\mathrm{NC}, K}^{2}+\left(\eta_{\mathrm{DF}, K}+\eta_{\mathrm{R}, K}\right)^{2}\right\} .
$$

Remark 6.13 (Constants in Theorem 6.12). We note that here a similar observation to that of Remark 6.9 holds true as well.

6.2.3. Fully computable estimates in the $L^{2}(\Omega)$-norm. The energy norm estimate of the previous section is designed to be used for the postprocessed approximation $\tilde{p}_{h}$ of Section 4.4 Using this result, we now derive $L^{2}(\Omega)$-norm estimates, first for $\tilde{p}_{h}$ and then for the original approximate potential $p_{h}$. As it will appear, however, these estimates are somewhat "less nice" than those of the previous section, as they in particular feature several, albeit known, constants in the leading terms; we do not find them optimal.

We first give an $L^{2}(\Omega)$-norm estimate for $\tilde{p}_{h}$, again in the most general setting possible:

Corollary 6.14 (A posteriori estimate for $\tilde{p}_{h}$ in the $L^{2}(\Omega)$-norm). Let $p$ be given by (1.2) and let $\tilde{p}_{h} \in W_{0}\left(\mathcal{T}_{h}\right)$ and $\mathbf{u}_{h} \in \mathbf{H}(\operatorname{div}, \Omega)$ such that $\nabla \cdot \mathbf{u}_{h}=P_{\Phi_{h}}(f)$ be arbitrary. Then

$$
\left\|p-\tilde{p}_{h}\right\|^{2} \leq \frac{C_{\mathrm{DF}}}{c_{\mathbf{S}, \Omega}} \sum_{K \in \mathcal{T}_{h}}\left\{\eta_{\mathrm{NC}, K}^{2}+\left(\eta_{\mathrm{DF}, K}+\eta_{\mathrm{R}, K}\right)^{2}\right\},
$$

where $\eta_{\mathrm{NC}, K}, \eta_{\mathrm{DF}, K}$, and $\eta_{\mathrm{R}, K}$ are given respectively by (6.7), (6.8), and (6.4).

Proof. This is immediate from Theorem 6.12, using the fact that $\left(p-\tilde{p}_{h}\right) \in W_{0}\left(\mathcal{T}_{h}\right)$, the discrete Friedrichs inequality (2.8), and (2.7).

We conclude this section by an $L^{2}(\Omega)$-norm estimate for $p_{h}$, following trivially from Corollary 6.14 by the triangle inequality; in practice, again $p_{h}$ and $\mathbf{u}_{h}$ are given by (1.5a)-1.5b) and $\tilde{p}_{h}$ by (4.2a)-4.2b) or (4.4a - 4.4b):

Corollary 6.15 (A posteriori estimate for $p_{h}$ in the $L^{2}(\Omega)$-norm). Let $p$ be given by (1.2) and let $p_{h} \in \Phi_{h}, \tilde{p}_{h} \in W_{0}\left(\mathcal{T}_{h}\right)$, and $\mathbf{u}_{h} \in \mathbf{H}(\operatorname{div}, \Omega)$ such that $\nabla \cdot \mathbf{u}_{h}=$ $P_{\Phi_{h}}(f)$ be arbitrary. Then

$$
\left\|p-p_{h}\right\| \leq\left\{\frac{C_{\mathrm{DF}}}{c_{\mathbf{S}, \Omega}} \sum_{K \in \mathcal{T}_{h}}\left\{\eta_{\mathrm{NC}, K}^{2}+\left(\eta_{\mathrm{DF}, K}+\eta_{\mathrm{R}, K}\right)^{2}\right\}\right\}^{\frac{1}{2}}+\left\|\tilde{p}_{h}-p_{h}\right\|,
$$

where $\eta_{\mathrm{NC}, K}, \eta_{\mathrm{DF}, K}$, and $\eta_{\mathrm{R}, K}$ are given respectively by (6.7), (6.8), and (6.4). 
6.3. Local efficiency. We prove here local efficiency of the a posteriori error estimators of Theorems 6.8 and 6.12 .

Theorem 6.16 (Local efficiency of estimators of Theorems 6.8 and 6.12). Let $f$ be piecewise polynomial of order $m$ and let $\mathbf{u}, p$ be given by (1.4a) $1.4 \mathrm{~b}$. Next, let $\mathcal{T}_{h}$ be shape-regular, let $\mathbf{u}_{h} \in \mathbf{H}(\operatorname{div}, \Omega)$ be such that $\nabla \cdot \mathbf{u}_{h}=P_{\Phi_{h}}(f)$, and $\tilde{p}_{h} \in \mathbb{R}_{n}\left(\mathcal{T}_{h}\right) \cap W_{0}\left(\mathcal{T}_{h}\right)$ for some $n \geq 1$. Finally, let the a posteriori error estimators $\eta_{\mathrm{P}, K}, \eta_{\mathrm{R}, K}, \eta_{\mathrm{NC}, K}$, and $\eta_{\mathrm{DF}, K}$ be given respectively by (6.3), (6.4), (6.7), and (6.8). Then

$$
\begin{aligned}
\eta_{\mathrm{P}, K} & \leq \eta_{\mathrm{DF}, K}+\eta_{\mathrm{NC}, K}, \\
\eta_{\mathrm{DF}, K} & \leq\left\|\mathbf{u}-\mathbf{u}_{h} \mid\right\|_{*, K}+\left\|p-\tilde{p}_{h}\right\|_{K}, \\
\eta_{\mathrm{NC}, K} & \leq C \sqrt{\frac{C_{\mathbf{S}, K}}{c_{\mathbf{S}, \mathcal{T}_{K}}}}\left\|p-\tilde{p}_{h}\right\| \|_{\mathcal{T}_{K}}, \\
\eta_{\mathrm{R}, K} & \leq \tilde{C} \sqrt{\frac{C_{\mathbf{S}, K}}{c_{\mathbf{S}, K}}}\left\|\mathbf{u}-\mathbf{u}_{h} \mid\right\|_{*, K},
\end{aligned}
$$

where the constant $C$ depends only on the space dimension $d$, the maximal polynomial degree $n$ of $\tilde{p}_{h}$, and the shape regularity parameter $\kappa_{\mathcal{T}}$ and $\tilde{C}$ depends only on $d$, the polynomial degree $m$ of $f$, and $\kappa_{\mathcal{T}}$.

Proof. For $\eta_{\mathrm{P}, K}$, we have

$$
\eta_{\mathrm{P}, K} \leq\left\|\mathbf{u}_{h}+\mathbf{S} \nabla \tilde{p}_{h}\right\|_{*, K}+\left\|\mathbf{S} \nabla \tilde{p}_{h}-\mathbf{S} \nabla\left(\mathcal{I}_{\mathrm{av}}\left(\tilde{p}_{h}\right)\right)\right\|_{*, K}=\eta_{\mathrm{DF}, K}+\eta_{\mathrm{NC}, K}
$$

by the triangle inequality. Similarly,

$$
\eta_{\mathrm{DF}, K} \leq\left|\left\|\mathbf{u}_{h}+\mathbf{S} \nabla p\right\|\left\|_{*, K}+|| \mathbf{S} \nabla p-\mathbf{S} \nabla \tilde{p}_{h}\right\|\right|_{*, K}=\left\|\left|\mathbf{u}-\mathbf{u}_{h}\right|\right\|_{*, K}+\left\||| p-\tilde{p}_{h} \mid\right\|_{K}
$$

by the triangle inequality and (2.10). Next, the inequality

$$
h_{\sigma}^{-\frac{1}{2}}\left\|\llbracket \tilde{p}_{h} \rrbracket\right\|_{\sigma} \leq C \sum_{L ; \sigma \in \mathcal{E}_{L}}\left\|\nabla\left(\tilde{p}_{h}-\varphi\right)\right\|_{L}
$$

was established in [3, Theorem 10] for $\tilde{p}_{h} \in W_{0}\left(\mathcal{T}_{h}\right)$, simplicial meshes, $\sigma \in \mathcal{E}_{h}^{\text {int }}$, and an arbitrary $\varphi \in H^{1}(\Omega)$. It generalizes easily to rectangular parallelepipeds and to the case $\sigma \in \mathcal{E}_{h}^{\text {ext }}$ and $\varphi \in H_{0}^{1}(\Omega)$; here $C$ depends only on $d$ and $\kappa_{\mathcal{T}}$. Thus for the nonconformity estimator we have

$$
\begin{aligned}
\eta_{\mathrm{NC}, K}^{2} & =\left\|\tilde{p}_{h}-\mathcal{I}_{\mathrm{av}}\left(\tilde{p}_{h}\right)\right\|_{K}^{2} \leq C C_{\mathbf{S}, K} \sum_{\sigma \in \tilde{\mathcal{E}}_{K}} h_{\sigma}^{-1}\left\|\llbracket \tilde{p}_{h} \rrbracket\right\|_{\sigma}^{2} \\
& \leq C C_{\mathbf{S}, K} \sum_{L \in \mathcal{T}_{K}}\left\|\nabla\left(p-\tilde{p}_{h}\right)\right\|_{L}^{2} \leq C \frac{C_{\mathbf{S}, K}}{c_{\mathbf{S}, \mathcal{T}_{K}}} \sum_{L \in \mathcal{T}_{K}}\left\|p-\tilde{p}_{h}\right\|_{L}^{2},
\end{aligned}
$$

using Lemma 2.1 and the above estimate, with $C$ depending only on $d, n$, and $\kappa_{\mathcal{T}}$. Finally,

$$
\left\|f-P_{\Phi_{h}}(f)\right\|_{K}=\left\|f-\nabla \cdot \mathbf{u}_{h}\right\|_{K} \leq C C_{\mathbf{S}, K}^{1 / 2} h_{K}^{-1} \mid\left\|\mathbf{u}-\mathbf{u}_{h}\right\| \|_{*, K}
$$

with $C$ depending only on $d, \kappa_{\mathcal{T}}$, and $m$ follows standardly by using the element bubble function, the equivalence of norms on finite-dimensional spaces, the definition (1.2) of the weak solution, the Green theorem, the Cauchy-Schwarz inequality, the definition (2.9) of the energy norm, and the inverse inequality; cf. [58] or [62, Lemma 7.6]. Note that we do not need $\mathbf{u}_{h}$ to be a polynomial and that 
$\nabla \cdot \mathbf{u}_{h}=P_{\Phi_{h}}(f)$ is a polynomial of maximal degree $m$ by the assumption on $f$. Hence the estimate for $\eta_{\mathrm{R}, K}$ follows.

6.4. Extensions. We present here two extensions of the previous results. First of all, following Bernardi and Verfürth [14] and Ainsworth [5] and using the averaging operator with diffusion tensor-dependent weights, one can obtain estimates robust with respect to inhomogeneities under the "monotonicity" assumption. Second, we show that our estimates are robust with respect to all inhomogeneities, anisotropies, polynomial degree, and mesh regularity for the error in the pair $\mathbf{u}_{h}$, $\mathcal{I}_{\text {av }}\left(\tilde{p}_{h}\right)$ considered as an approximate solution.

6.4.1. Estimates robust with respect to inhomogeneities under the "monotonicity" assumption. With the notation of Section 2.3, let

$$
\mathcal{I}_{\text {av }, \mathbf{S}}\left(\varphi_{h}\right)(V)=\left.\frac{1}{\sum_{K \in \mathcal{T}_{V}} C_{\mathbf{S}, K}^{1 / 2}} \sum_{K \in \mathcal{T}_{V}} C_{\mathbf{S}, K}^{\frac{1}{2}} \varphi_{h}\right|_{K}(V) .
$$

Then all the estimates of Sections 6.1 and 6.2 hold true with $\mathcal{I}_{\text {av }}$ replaced by $\mathcal{I}_{\text {av }, \mathbf{S}}$. Clearly, the difference between $\mathcal{I}_{\mathrm{av}}$ and $\mathcal{I}_{\mathrm{av}, \mathbf{S}}$ is the use of the diffusion tensordependent weights in the latter. We first make the following assumption (cf. [14, Hypothesis 2.7]):

Assumption (C) (Monotonicity of the distribution of $C_{\mathbf{S}, K}$ ). For any two elements $L, M \in \mathcal{T}_{h}$ which share at least one point, there exists a connected path passing from $L$ to $M$ through element sides such that the function $C_{\mathbf{S}, K}$ is monotone along this path.

We then have the following result:

Theorem 6.17 (Local efficiency robust with respect to inhomogeneities under Assumption (C)). Let all the assumptions of Theorem 6.16 hold, with $\mathcal{I}_{\mathrm{av}}$ replaced by $\mathcal{I}_{\mathrm{av}, \mathbf{S}}$. Next, let Assumption $(C)$ hold. Then

$$
\begin{aligned}
\eta_{\mathrm{P}, K} & \leq \eta_{\mathrm{DF}, K}+\eta_{\mathrm{NC}, K}, \\
\eta_{\mathrm{DF}, K} & \leq\left\|\mathbf{u}-\mathbf{u}_{h} \mid\right\|_{*, K}+\left\|p-\tilde{p}_{h}\right\|_{K}, \\
\eta_{\mathrm{NC}, K} & \leq C \max _{K \in \mathcal{T}_{K}} \sqrt{\frac{C_{\mathbf{S}, K}}{c_{\mathbf{S}, K}}}\left\|p-\tilde{p}_{h}\right\| \|_{\mathcal{T}_{K}}, \\
\eta_{\mathrm{R}, K} & \leq \tilde{C} \sqrt{\frac{C_{\mathbf{S}, K}}{c_{\mathbf{S}, K}}}\left\|\mathbf{u}-\mathbf{u}_{h}\right\| \|_{*, K},
\end{aligned}
$$

where the constant $C$ depends only on the space dimension $d$, the maximal polynomial degree $n$ of $\tilde{p}_{h}$, and the shape regularity parameter $\kappa_{\mathcal{T}}$ and $\tilde{C}$ depends only on $d$, the polynomial degree $m$ of $f$, and $\kappa_{\mathcal{T}}$.

Unfortunately, for the above robustness result, the "monotonicity" assumption is crucial. Consequently, some of the most interesting cases with a checkerboard distribution of values of the diffusion coefficient, inducing a singularity, are excluded. For conforming discretizations, estimates robust in all cases are presented in 63. The generalization to the nonconforming case represents an ongoing work. 
6.4.2. Estimates robust with respect to inhomogeneities, anisotropies, polynomial degree, and mesh regularity for flux- and potential-conforming approximations. Combining Theorems 6.8 and 6.12 for the upper bound and the triangle inequality and the estimate for $\eta_{\mathrm{R}, K}$ from Theorem 6.16 for the local efficiency, we can state the following result:

Theorem 6.18 (Optimal a posteriori error estimate for flux- and potential-conforming approximations). Let $\mathbf{u}, p$ be given by (1.4a - 1.4b and let $\mathbf{u}_{h} \in \mathbf{H}(\operatorname{div}, \Omega)$ such that $\nabla \cdot \mathbf{u}_{h}=P_{\Phi_{h}}(f), \tilde{p}_{h} \in H^{1}\left(\mathcal{T}_{h}\right)$, and $s_{h} \in H_{0}^{1}(\Omega)$ be arbitrary. Next, let the a posteriori error estimators $\eta_{\mathrm{P}, K}, \eta_{\mathrm{R}, K}, \eta_{\mathrm{NC}, K}$, and $\eta_{\mathrm{DF}, K}$ be given respectively by (6.3), (6.4), (6.7), and (6.8), with $\mathcal{I}_{\mathrm{av}}\left(\tilde{p}_{h}\right)$ replaced by $s_{h}$. Then

$$
\left\|\mathbf{u}-\mathbf{u}_{h}\left|\left\|_{*}^{2}+\right\|\right| p-s_{h}||^{2} \leq \sum_{K \in \mathcal{T}_{h}}\left\{\eta_{\mathrm{P}, K}^{2}+\eta_{\mathrm{R}, K}^{2}+\left(\eta_{\mathrm{P}, K}+\eta_{\mathrm{R}, K}\right)^{2}\right\}\right.
$$

and

$$
\eta_{\mathrm{P}, K} \leq\left\|\mathbf{u}-\mathbf{u}_{h}\right\|_{*, K}+\|\| p-s_{h} \|_{K}
$$

Similarly,

$$
\begin{aligned}
\|\| \mathbf{u}-\mathbf{u}_{h}\left|\left\|_{*}^{2}+\right\| p-\tilde{p}_{h}\right|\left\|^{2}+\right\| p-s_{h} \|^{2} \leq & \sum_{K \in \mathcal{T}_{h}}\left\{\eta_{\mathrm{P}, K}^{2}+\eta_{\mathrm{R}, K}^{2}+\left(\eta_{\mathrm{P}, K}+\eta_{\mathrm{R}, K}\right)^{2}\right. \\
& \left.+\eta_{\mathrm{NC}, K}^{2}+\left(\eta_{\mathrm{DF}, K}+\eta_{\mathrm{R}, K}\right)^{2}\right\}
\end{aligned}
$$

and

$$
\begin{aligned}
\eta_{\mathrm{P}, K} & \leq\left\|\mathbf{u}-\mathbf{u}_{h}\right\|_{*, K}+\left\||| p-s_{h} \mid\right\|_{K}, \\
\eta_{\mathrm{DF}, K} & \leq\left\|\mathbf{u}-\mathbf{u}_{h}\right\|_{*, K}+\left\||| p-\tilde{p}_{h} \mid\right\|_{K}, \\
\eta_{\mathrm{NC}, K} & \leq\left\|\left|\left\|p-\tilde{p}_{h}\left|\left\|_{K}+\right\|\right|\left|p-s_{h}\right|\right\|\right|_{K} .\right.
\end{aligned}
$$

Finally, the residual estimators $\eta_{\mathrm{R}, K}$ may represent a higher-order term; see Remark 6.4. In any case, when $f$ is piecewise polynomial of order $m$ and $\mathcal{T}_{h}$ shaperegular, then

$$
\eta_{\mathrm{R}, K} \leq \tilde{C} \sqrt{\frac{C_{\mathbf{S}, K}}{c_{\mathbf{S}, K}}}\left\|\mathbf{u}-\mathbf{u}_{h}\right\|_{*, K}
$$

where $\tilde{C}$ depends only on $d$, the polynomial degree $m$ of $f$, and $\kappa_{\mathcal{T}}$.

Remark 6.19 (Theorem 6.18). Theorem 6.18 shows that, possibly up to the residual term, a posteriori error estimates robust with respect to all the diffusion tensor $\mathbf{S}$, the space dimension $d$, the maximal polynomial degree of $\mathbf{u}_{h}, s_{h}$, and $\tilde{p}_{h}$, and the mesh shape regularity can easily be given when the pair $\mathbf{u}_{h}, \mathcal{I}_{\text {av }}\left(\tilde{p}_{h}\right)$ (and not the pair $\left.\mathbf{u}_{h}, \tilde{p}_{h}\right)$ or the triple $\mathbf{u}_{h}, \mathcal{I}_{\text {av }}\left(\tilde{p}_{h}\right), \tilde{p}_{h}$ is considered as an approximate solution. Moreover, a maximal overestimation factor (effectivity index) is guaranteed. Concerning the residual term, the estimates can be given for $\left\|\left|\mathbf{u}-\mathbf{u}_{h}\right|\right\|_{* \text {, div }}$ as in Theorem 6.8. This is in agreement with the results of Repin et al. [55]. Basically, giving optimal a posteriori error estimates for approximations which are both fluxand potential-conforming is trivial. 


\section{Complements ON MiXed Finite ElEMENT Methods}

We give here some complements on mixed finite element methods which seem to be new. We start by showing that under the assumption the source function $f$ belongs to the space $\Phi_{h}$, some orthogonal projection relations are valid in the mixed finite element method, parallel and complementary to the conforming finite element method. We next show that mixed finite element approximate solutions are directly equal to or very close to some generalized weak solutions.

7.1. Orthogonal projection properties. We first give the following characterization, valid for any mixed finite element scheme.

Theorem 7.1 (Vector orthogonal projection property). Let $f \in \Phi_{h}$, let $p$ be given by (1.4a) - 1.4b), and let $\mathbf{u}_{h} \in \mathbf{H}(\operatorname{div}, \Omega)$ such that $\nabla \cdot \mathbf{u}_{h}=f$ is arbitrary. Then

$$
\left\|\mathbf{u}_{h}+\mathbf{S} \nabla p\right\|\left\|_{*}=\inf _{s \in H_{0}^{1}(\Omega)}\right\| \mid \mathbf{u}_{h}+\mathbf{S} \nabla s \|_{*},
$$

or, equivalently,

$$
\mathcal{A}\left(\mathbf{S} \nabla p+\mathbf{u}_{h}, \mathbf{S} \nabla \varphi\right)=0 \quad \forall \varphi \in H_{0}^{1}(\Omega) .
$$

Proof. Property (7.1) follows immediately from (6.1) under the assumption $f \in \Phi_{h}$. Then (7.2) is standard; alternatively, let $\varphi \in H_{0}^{1}(\Omega)$ and note that

$$
\mathcal{A}\left(-\mathbf{u}_{h}, \mathbf{S} \nabla \varphi\right)=\left(-\mathbf{u}_{h}, \nabla \varphi\right)=(f, \varphi)
$$

by (2.3), the Green theorem, and the assumption $\nabla \cdot \mathbf{u}_{h}=f$. Now put $\mathbf{w}=$ $\mathbf{u}_{h}$ in Theorem 3.3 and notice that the function $\psi$ from (3.1) coincides with $p$. Consequently, (7.2) follows from (3.4).

Remark 7.2 (Vector orthogonal projection property). In the conforming finite element method for (1.1a)-1.1b), the approximate solution $q_{h} \in X_{h}$ with $X_{h}:=$ $\mathbb{R}_{k}\left(\mathcal{T}_{h}\right) \cap H_{0}^{1}(\Omega)$ is characterized by

$$
\mathcal{B}\left(q_{h}, \varphi_{h}\right)=\left(f, \varphi_{h}\right) \quad \forall \varphi_{h} \in X_{h}
$$

and satisfies

$$
\begin{aligned}
\left\|p-q_{h}\right\| \| & =\inf _{s_{h} \in X_{h}}\left\|p-s_{h}\right\| \|, \\
\mathcal{B}\left(p-q_{h}, \varphi_{h}\right) & =0 \quad \forall \varphi_{h} \in X_{h} .
\end{aligned}
$$

This means that it is the $H_{0}^{1}(\Omega)$-orthogonal projection of the exact potential $p$ onto $X_{h}$ with respect to the scalar product $\mathcal{B}(\cdot, \cdot)$ (and the associated scalar energy norm (2.7) ). We denote this projection by $P_{X_{h}}$. Theorem 7.1 says that in the mixed finite element method, under the condition that $f \in \Phi_{h}$, the exact flux $\mathbf{u}=-\mathbf{S} \nabla p$ is the $\mathbf{L}^{2}(\Omega)$-orthogonal projection of the approximate flux $\mathbf{u}_{h}$ onto $\mathbf{S} \nabla H_{0}^{1}(\Omega)$ with respect to the scalar product $\mathcal{A}(\cdot, \cdot)$ (and the associated vector energy norm (2.9) ). Note the parallel and also the exchange of the roles between the exact and approximate solutions: in the conforming finite element method, the approximate solution is the orthogonal projection of the exact one, whereas in the mixed finite element case, the exact solution is the orthogonal projection of the approximate one.

The following characterization is only valid in the lowest-order Raviart-ThomasNédélec case: 


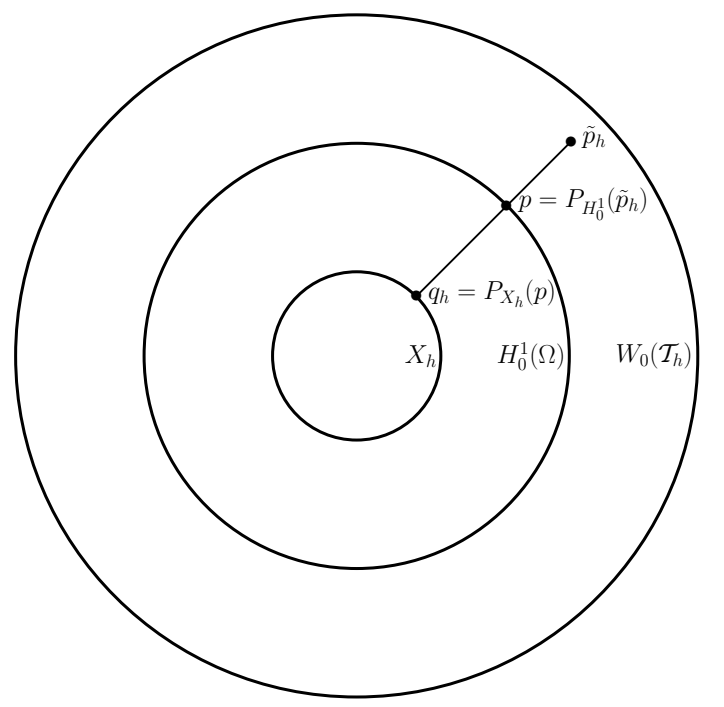

Figure 1. Graphical visualization of the relations between the postprocessed lowest-order mixed finite solution $\tilde{p}_{h}$, the weak solution $p$, and the conforming finite element solution $q_{h}$ when $f \in$ $\Phi_{h}$.

Theorem 7.3 (Scalar orthogonal projection property). Let Assumption (A) hold, let $f \in \Phi_{h}$, and let $p$ be given by (1.4a) -(1.4b), $\mathbf{u}_{h}, p_{h}$ by (1.5a)-(1.5b), and $\tilde{p}_{h}$ by (4.2a) - (4.2b). Then

$$
\left\|\left|p-\tilde{p}_{h}\right|\right\|=\inf _{s \in H_{0}^{1}(\Omega)}\left|\left\|\tilde{p}_{h}-s \mid\right\|,\right.
$$

or, equivalently,

$$
\mathcal{B}\left(p-\tilde{p}_{h}, \varphi\right)=0 \quad \forall \varphi \in H_{0}^{1}(\Omega) .
$$

Proof. This is immediate from (7.1) and (7.2) using (1.3a), (4.2a), and (2.10).

Remark 7.4 (Scalar orthogonal projection property). Under the assumptions of Theorem [7.3] the exact potential $p$ is the $W_{0}\left(\mathcal{T}_{h}\right)$-orthogonal projection of the approximate postprocessed potential $\tilde{p}_{h}$ onto $H_{0}^{1}(\Omega)$ with respect to the scalar product $\mathcal{B}(\cdot, \cdot)$ (and the associated scalar energy norm (2.7)). We denote this projection by $P_{H_{0}^{1}}$. Here, the parallel to the conforming finite element method is even stronger, compare it with Remark 7.2. The situation is graphically illustrated in Figure 1.

7.2. Generalized weak solutions and mixed finite elements. We develop here the ideas of [62, Section 5.4] on the relation between mixed finite element approximate solutions and certain generalized weak solutions. For some results comparing the mixed and (generalized) finite element approximate solutions, we refer to Babuška and Osborn [11] and Falk and Osborn [38].

By a generalized weak solution, we understand a function $\tilde{p} \in W_{h}\left(\mathcal{T}_{h}\right)$ such that

$$
(\mathbf{S} \nabla \tilde{p}, \nabla \varphi)=(f, \varphi) \quad \forall \varphi \in W_{h}\left(\mathcal{T}_{h}\right) .
$$

Note that (2.7), (2.11a), and the discrete Friedrichs inequality (2.8) ensure the existence and uniqueness of the solution of (7.3). This generalized weak solution is 
dependent on the given mesh $\mathcal{T}_{h}$ and also on the normal components of the space $\mathbf{V}_{h}$ by the definition (2.1b) of the space $W_{h}\left(\mathcal{T}_{h}\right)$. Note also that $H_{0}^{1}(\Omega) \subset W_{h}\left(\mathcal{T}_{h}\right)$, whence the term "generalized".

Theorem 7.5 (A posteriori estimates for the generalized weak solutions). Let $\tilde{p}$ be given by (7.3), $\tilde{\mathbf{u}}$ by $\tilde{\mathbf{u}}:=-\mathbf{S} \nabla \tilde{p}, \mathbf{u}_{h}, p_{h}$ by (1.5a) (1.5b), and $\tilde{p}_{h}$ by (4.2a) (4.2b) or (4.4a) - (4.4b). Then

$$
\begin{aligned}
\left\|\left|\tilde{\mathbf{u}}-\mathbf{u}_{h}\right|\right\|_{*}^{2} & \leq \sum_{K \in \mathcal{T}_{h}}\left(\eta_{\mathrm{DF}, K}^{2}+\eta_{\mathrm{R}, K}^{2}\right), \\
\left\|\tilde{p}-\tilde{p}_{h} \mid\right\|^{2} & \leq \sum_{K \in \mathcal{T}_{h}}\left(\eta_{\mathrm{DF}, K}+\eta_{\mathrm{R}, K}\right)^{2} .
\end{aligned}
$$

where the diffusive flux estimator $\eta_{\mathrm{DF}, K}$ is given by (6.8) and the residual estimator $\eta_{\mathrm{R}, K}$ by (6.4),

Proof. By replacing $H_{0}^{1}(\Omega)$ by $W_{h}\left(\mathcal{T}_{h}\right)$ in Theorem 3.3. putting $\mathbf{v}=\tilde{\mathbf{u}}, \mathbf{w}=\mathbf{u}_{h}$, and using (3.3), one comes to the equivalent of (3.2)-(3.3) in the form

$$
\left\|\tilde{\mathbf{u}}-\mathbf{u}_{h}\right\|_{*}^{2}=\inf _{s \in W_{h}\left(\mathcal{T}_{h}\right)}\left\|\mathbf{u}_{h}+\mathbf{S} \nabla s\right\|_{*}^{2}+\mathcal{A}\left(\tilde{\mathbf{u}}-\mathbf{u}_{h}, \frac{\tilde{\mathbf{u}}+\mathbf{S} \nabla \psi}{\|\tilde{\mathbf{u}}+\mathbf{S} \nabla \psi\|_{*}}\right)^{2} .
$$

We next put $\varphi:=(\tilde{p}-\psi) /\|\tilde{p}-\psi\| \mid \in W_{h}\left(\mathcal{T}_{h}\right)$ and rewrite the second term of the above expression as $\mathcal{A}\left(\tilde{\mathbf{u}}-\mathbf{u}_{h},-\mathbf{S} \nabla \varphi\right)$, employing $\tilde{\mathbf{u}}=-\mathbf{S} \nabla \tilde{p}$ and (2.10). Next, by (2.3) and the definition of the generalized weak solution (7.3),

$$
\mathcal{A}(\tilde{\mathbf{u}},-\mathbf{S} \nabla \varphi)=(f, \varphi),
$$

whereas

$$
\begin{aligned}
\mathcal{A}\left(\mathbf{u}_{h},-\mathbf{S} \nabla \varphi\right) & =-\left(\mathbf{u}_{h}, \nabla \varphi\right)=\sum_{K \in \mathcal{T}_{h}}\left\{\left(\nabla \cdot \mathbf{u}_{h}, \varphi\right)_{K}-\left\langle\mathbf{u}_{h} \cdot \mathbf{n}, \varphi\right\rangle_{\partial K}\right\} \\
& =\left(P_{\Phi_{h}}(f), \varphi\right)
\end{aligned}
$$

by (2.3), the Green theorem, the fact that $\mathbf{u}_{h} \in \mathbf{V}_{h}$ and $\varphi \in W_{h}\left(\mathcal{T}_{h}\right)$, and (1.5b). Note the importance of the definition (2.1b) of the space $W_{h}\left(\mathcal{T}_{h}\right)$, by which the term $\sum_{K \in \mathcal{T}_{h}}\left\langle\mathbf{u}_{h} \cdot \mathbf{n}, \varphi\right\rangle_{\partial K}=\sum_{\sigma \in \mathcal{E}_{h}}\left\langle\mathbf{u}_{h} \cdot \mathbf{n}, \llbracket \varphi \rrbracket\right\rangle_{\sigma}$ disappears. Hence

$$
\mathcal{A}\left(\tilde{\mathbf{u}}-\mathbf{u}_{h},-\mathbf{S} \nabla \varphi\right)=\left(f-P_{\Phi_{h}}(f), \varphi\right) .
$$

Estimating this term exactly as in the proof of Theorem 6.1 and putting $s=\tilde{p}_{h}$, the estimate for $\tilde{\mathbf{u}}-\mathbf{u}_{h}$ follows.

Similarly, as in the vector case, instead of (6.6), in the present setting one gets

$$
\left\|\tilde{p}-\tilde{p}_{h}\left|\left\|^{2} \leq \inf _{s \in W_{h}\left(\mathcal{T}_{h}\right)}\right\| \tilde{p}_{h}-s \|\right|^{2}+\sup _{\varphi \in W_{h}\left(\mathcal{T}_{h}\right),\|\varphi\| \|=1}\left(\left(f-\nabla \cdot \mathbf{u}_{h}, \varphi\right)-\left(\mathbf{S} \nabla \tilde{p}_{h}+\mathbf{u}_{h}, \nabla \varphi\right)\right)^{2} .\right.
$$

As the first term disappears since $\tilde{p}_{h} \in W_{h}\left(\mathcal{T}_{h}\right)$, the estimate for $\tilde{p}-\tilde{p}_{h}$ follows by the Cauchy-Schwarz inequality.

Remark 7.6 (A posteriori estimates for the generalized weak solutions). Note that the essential difference of the estimates of Theorem 7.5 and of those of Theorems 6.8 and 6.12 are that the nonconformity estimator $\eta_{\mathrm{NC}, K}$ given by (6.7) and the potential estimator $\eta_{\mathrm{P}, K}$ given by (6.3), the two estimators penalizing the nonconformity in $\tilde{p}_{h}$ through the introduction of the averaging $\mathcal{I}_{\text {av }}\left(\tilde{p}_{h}\right)$, are not present, since the generalized solution $\tilde{p}$ is itself in the space $W_{h}\left(\mathcal{T}_{h}\right)$ as $\tilde{p}_{h}$. Note also that under Assumption (A), the diffusive flux estimators $\eta_{\mathrm{DF}, K}$ vanish, whereas for $f \in \Phi_{h}$, 
the residual estimators $\eta_{\mathrm{R}, K}$ vanish. Thus in the lowest-order Raviart-ThomasNédélec case and for elementwise constant $f, \tilde{p}=\tilde{p}_{h}$ (and $\tilde{\mathbf{u}}=\mathbf{u}_{h}$ ). We refer to 62, Sections 5.4 and 5.6] for a more detailed discussion of this special case.

The proof of the following theorem is straightforward, using the same techniques as those in the proof of Theorem 6.16.

Theorem 7.7 (Local efficiency of estimators of Theorem 7.5). Let the assumptions of Theorem 7.5 be verified. Then

$$
\eta_{\mathrm{DF}, K} \leq||\left|\tilde{\mathbf{u}}-\mathbf{u}_{h}\right|\left\|_{*, K}+\right\| \mid \tilde{p}-\tilde{p}_{h} \|_{K} .
$$

Moreover, the residual estimators $\eta_{\mathrm{R}, K}$ may represent a higher-order term; see Remark 6.4. In any case, when $f$ is piecewise polynomial of order $m$ and $\mathcal{T}_{h}$ shaperegular, then

$$
\eta_{\mathrm{R}, K} \leq \tilde{C} \sqrt{\frac{C_{\mathbf{S}, K}}{c_{\mathbf{S}, K}}}\left\|\tilde{\mathbf{u}}-\mathbf{u}_{h}\right\|_{*, K},
$$

where $\tilde{C}$ depends only on $d$, the polynomial degree $m$ of $f$, and $\kappa_{\mathcal{T}}$.

Remark 7.8 (Local efficiency of estimators of Theorem 7.5). Note that, possibly up to the residual term, the a posteriori error estimate of Theorem 7.5 is, according to Theorem 7.7, robust with respect to all the diffusion tensor $\mathbf{S}$, the space dimension $d$, the maximal polynomial degree $n$ of $\tilde{p}_{h}$, and the mesh shape regularity.

\section{REFERENCES}

1. Ivar Aavatsmark, Tor Barkve, Øistein Bøe, and Trond Mannseth, Discretization on unstructured grids for inhomogeneous, anisotropic media. I. Derivation of the methods, SIAM J. Sci. Comput. 19 (1998), no. 5, 1700-1716. MR.1618761 (99f:65138)

2. Boujemâa Achchab, Abdellatif Agouzal, Jacques Baranger, and Jean-François Maître, Estimateur d'erreur a posteriori hiérarchique. Application aux éléments finis mixtes, Numer. Math. 80 (1998), no. 2, 159-179. MR1645037 (2000d:65092)

3. Yves Achdou, Christine Bernardi, and Frédéric Coquel, A priori and a posteriori analysis of finite volume discretizations of Darcy's equations, Numer. Math. 96 (2003), no. 1, 17-42. MR2018789 (2005d:65179)

4. Robert A. Adams, Sobolev spaces, Academic Press [A subsidiary of Harcourt Brace Jovanovich, Publishers], New York-London, 1975, Pure and Applied Mathematics, Vol. 65. MR0450957 (56:9247)

5. Mark Ainsworth, Robust a posteriori error estimation for nonconforming finite element approximation, SIAM J. Numer. Anal. 42 (2005), no. 6, 2320-2341. MR2139395

6. Mark Ainsworth, A posteriori error estimation for lowest order Raviart-Thomas mixed finite elements, SIAM J. Sci. Comput. 30 (2007), no. 1, 189-204. MR2377438 (2008m:65308)

7. Ana Alonso, Error estimators for a mixed method, Numer. Math. 74 (1996), no. 4, 385-395. MR.1414415 (97g:65212)

8. Todd Arbogast and Zhangxin Chen, On the implementation of mixed methods as nonconforming methods for second-order elliptic problems, Math. Comp. 64 (1995), no. 211, 943-972. MR.1303084 (95k:65102)

9. Douglas N. Arnold and Franco Brezzi, Mixed and nonconforming finite element methods: implementation, postprocessing and error estimates, RAIRO Modél. Math. Anal. Numér. 19 (1985), no. 1, 7-32. MR813687 (87g:65126)

10. Ivo Babuška, John E. Osborn, and Juhanni Pitkäranta, Analysis of mixed methods using mesh dependent norms, Math. Comp. 35 (1980), no. 152, 1039-1062. MR.583486 (81m:65166)

11. Ivo Babuška and John E. Osborn, Generalized finite element methods: their performance and their relation to mixed methods, SIAM J. Numer. Anal. 20 (1983), no. 3, 510-536. MR701094 (84h:65076)

12. Ivo Babuška, Error-bounds for finite element method, Numer. Math. 16 (1971), 322-333. MR 0288971(44:6166) 
13. Mario Bebendorf, A note on the Poincaré inequality for convex domains, Z. Anal. Anwendungen 22 (2003), no. 4, 751-756. MR2036927 (2004k:26025)

14. Christine Bernardi and Rüdiger Verfürth, Adaptive finite element methods for elliptic equations with non-smooth coefficients, Numer. Math. 85 (2000), no. 4, 579-608. MR1771781 (2001e:65177)

15. Dietrich Braess and Rüdiger Verfürth, A posteriori error estimators for the Raviart-Thomas element, SIAM J. Numer. Anal. 33 (1996), no. 6, 2431-2444. MR:1427472 (97m:65201)

16. James H. Bramble and Jinchao Xu, A local post-processing technique for improving the accuracy in mixed finite-element approximations, SIAM J. Numer. Anal. 26 (1989), no. 6, 1267-1275. MR 1025087 (90m:65193)

17. Franco Brezzi, On the existence, uniqueness and approximation of saddle-point problems arising from Lagrangian multipliers, Rev. Française Automat. Informat. Recherche Opérationnelle Sér. Rouge 8 (1974), no. R-2, 129-151. MR0365287 (51:1540)

18. Franco Brezzi, Jim Douglas, Jr., Ricardo Durán, and Michel Fortin, Mixed finite elements for second order elliptic problems in three variables, Numer. Math. 51 (1987), no. 2, 237-250. MR890035 (88f:65190)

19. Franco Brezzi, Jim Douglas, Jr., and L. Donatella Marini, Two families of mixed finite elements for second order elliptic problems, Numer. Math. 47 (1985), no. 2, 217-235. MR799685 (87g:65133)

20. Franco Brezzi and Michel Fortin, Mixed and hybrid finite element methods, Springer Series in Computational Mathematics, vol. 15, Springer-Verlag, New York, 1991. MR.1115205 (92d:65187)

21. Franco Brezzi, Konstantin Lipnikov, and Mikhail Shashkov, Convergence of the mimetic finite difference method for diffusion problems on polyhedral meshes, SIAM J. Numer. Anal. 43 (2005), no. 5, 1872-1896. MR2192322 (2006j:65311)

22. Erik Burman and Alexandre Ern, Continuous interior penalty hp-finite element methods for advection and advection-diffusion equations, Math. Comp. 76 (2007), no. 259, 1119-1140. MR 2299768

23. Carsten Carstensen, A posteriori error estimate for the mixed finite element method, Math. Comp. 66 (1997), no. 218, 465-476. MR1408371 (98a:65162)

24. Carsten Carstensen and Sören Bartels, Each averaging technique yields reliable a posteriori error control in FEM on unstructured grids. I. Low order conforming, nonconforming, and mixed FEM, Math. Comp. 71 (2002), no. 239, 945-969. MR.1898741(2003e:65212)

25. Zhangxin Chen, Analysis of mixed methods using conforming and nonconforming finite element methods, RAIRO Modél. Math. Anal. Numér. 27 (1993), no. 1, 9-34. MR1204626 (94c:65132)

26. Equivalence between and multigrid algorithms for nonconforming and mixed methods for second-order elliptic problems, East-West J. Numer. Math. 4 (1996), no. 1, 1-33. MR 1393063 (98c:65184)

27. So-Hsiang Chou, Do Y. Kwak, and Kwang Y. Kim, A general framework for constructing and analyzing mixed finite volume methods on quadrilateral grids: the overlapping covolume case, SIAM J. Numer. Anal. 39 (2001), no. 4, 1170-1196. MR1870838 (2002j:65109)

28. Bernardo Cockburn and Jayadeep Gopalakrishnan, A characterization of hybridized mixed methods for second order elliptic problems, SIAM J. Numer. Anal. 42 (2004), no. 1, 283-301. MR2051067 (2005e:65183)

29. tion, Math. Comp. 74 (2005), no. 252, 1653-1677. MR.2164091(2006e:65215)

30. Jim Douglas, Jr. and Jean E. Roberts, Mixed finite element methods for second order elliptic problems, Mat. Apl. Comput. 1 (1982), no. 1, 91-103. MR667620 (84b:65111)

31. Global estimates for mixed methods for second order elliptic equations, Math. Comp. 44 (1985), no. 169, 39-52. MR771029 (86b:65122)

32. Jérôme Droniou and Robert Eymard, A mixed finite volume scheme for anisotropic diffusion problems on any grid, Numer. Math. 105 (2006), no. 1, 35-71. MR.2257385

33. Ricardo Durán and Claudio Padra, An error estimator for nonconforming approximations of a nonlinear problem, Finite element methods (Jyväskylä, 1993), Lecture Notes in Pure and Appl. Math., vol. 164, Dekker, New York, 1994, pp. 201-205. MR.1299990 
34. Linda El Alaoui and Alexandre Ern, Residual and hierarchical a posteriori error estimates for nonconforming mixed finite element methods, M2AN Math. Model. Numer. Anal. 38 (2004), no. 6, 903-929. MR2108938

35. Alexandre Ern, Annette F. Stephansen, and Martin Vohralík, Guaranteed and robust discontinuous Galerkin a posteriori error estimates for convection-diffusion-reaction problems, J. Comput. Appl. Math. 234 (2010), no. 1, 114-130.

36. Robert Eymard, Thierry Gallouët, and Raphà̀le Herbin, Finite volume methods, Handbook of Numerical Analysis, Vol. VII, North-Holland, Amsterdam, 2000, pp. 713-1020. MR.1804748 (2002e:65138)

37. Robert Eymard, Danielle Hilhorst, and Martin Vohralík, A combined finite volume-nonconforming/mixed-hybrid finite element scheme for degenerate parabolic problems, Numer. Math. 105 (2006), no. 1, 73-131. MR2257386(2007i:76066)

38. Richard S. Falk and John E. Osborn, Remarks on mixed finite element methods for problems with rough coefficients, Math. Comp. 62 (1994), no. 205, 1-19. MR1203735 (94c:65136)

39. Ronald H. W. Hoppe and Barbara I. Wohlmuth, Adaptive multilevel techniques for mixed finite element discretizations of elliptic boundary value problems, SIAM J. Numer. Anal. 34 (1997), no. 4, 1658-1681. MR.1461801 (98e:65095)

40. Ohannes A. Karakashian and Frédéric Pascal, A posteriori error estimates for a discontinuous Galerkin approximation of second-order elliptic problems, SIAM J. Numer. Anal. 41 (2003), no. 6, 2374-2399. MR2034620 (2005d:65192)

41. Kwang Y. Kim, A posteriori error analysis for locally conservative mixed methods, Math. Comp. 76 (2007), no. 257, 43-66. MR2261011

42. Robert Kirby, Residual a posteriori error estimates for the mixed finite element method, Comput. Geosci. 7 (2003), no. 3, 197-214. MR2007578 (2004h:65112)

43. Mats G. Larson and Axel Målqvist, A posteriori error estimates for mixed finite element approximations of elliptic problems, Numer. Math. 108 (2008), no. 3, 487-500. MR2365826

44. Carlo Lovadina and Rolf Stenberg, Energy norm a posteriori error estimates for mixed finite element methods, Math. Comp. 75 (2006), no. 256, 1659-1674. MR2240629

45. L. Donatella Marini and Paola Pietra, An abstract theory for mixed approximations of second order elliptic problems, Mat. Apl. Comput. 8 (1989), no. 3, 219-239. MR:1067287|(91h:65177)

46. L. Donatella Marini, An inexpensive method for the evaluation of the solution of the lowest order Raviart-Thomas mixed method, SIAM J. Numer. Anal. 22 (1985), no. 3, 493-496. MR787572 (86g:65214)

47. Jean-Claude Nédélec, Mixed finite elements in $\mathbb{R}^{3}$, Numer. Math. 35 (1980), no. 3, 315-341. MR592160 (81k:65125)

48. Serge Nicaise and Emmanuel Creusé, Isotropic and anisotropic a posteriori error estimation of the mixed finite element method for second order operators in divergence form, Electron. Trans. Numer. Anal. 23 (2006), 38-62. MR2268551

49. Lawrence E. Payne and Hans F. Weinberger, An optimal Poincaré inequality for convex domains, Arch. Rational Mech. Anal. 5 (1960), 286-292. MR0117419 (22:8198)

50. Gergina V. Pencheva, Martin Vohralík, Mary F. Wheeler, and Tim Wildey, Robust a posteriori error control and adaptivity for multiscale, multinumerics, and mortar coupling, Preprint R10015, Laboratoire Jacques-Louis Lions, HAL Preprint 00467738, Submitted for publication, 2010.

51. William Prager and John L. Synge, Approximations in elasticity based on the concept of function space, Quart. Appl. Math. 5 (1947), 241-269. MR.0025902 (10,81b)

52. Alfio Quarteroni and Alberto Valli, Numerical approximation of partial differential equations, Springer Series in Computational Mathematics, vol. 23, Springer-Verlag, Berlin, 1994. MR.1299729 (95i:65005)

53. Pierre-Arnaud Raviart and Jean-Marie Thomas, A mixed finite element method for 2nd order elliptic problems, Mathematical aspects of finite element methods (Proc. Conf., Consiglio Naz. delle Ricerche (C.N.R.), Rome, 1975), Springer, Berlin, 1977, pp. 292-315. Lecture Notes in Math., Vol. 606. MR0483555 (58:3547)

54. Sergey I. Repin and Anton Smolianski, Functional-type a posteriori error estimates for mixed finite element methods, Russian J. Numer. Anal. Math. Modelling 20 (2005), no. 4, 365-382. MR2161108(2006c:65108) 
55. Sergey I. Repin, Stefan Sauter, and Anton Smolianski, Two-sided a posteriori error estimates for mixed formulations of elliptic problems, SIAM J. Numer. Anal. 45 (2007), no. 3, 928-945. MR 2318795 (2009c:65275)

56. Jean E. Roberts and Jean-Marie Thomas, Mixed and hybrid methods, Handbook of Numerical Analysis, Vol. II, North-Holland, Amsterdam, 1991, pp. 523-639. MR.1115239

57. Rolf Stenberg, Postprocessing schemes for some mixed finite elements, RAIRO Modél. Math. Anal. Numér. 25 (1991), no. 1, 151-167. MR1086845 (92a:65303)

58. R. Verfürth, A review of a posteriori error estimation and adaptive mesh-refinement techniques, Teubner-Wiley, Stuttgart, 1996.

59. Martin Vohralík, On the discrete Poincaré-Friedrichs inequalities for nonconforming approximations of the Sobolev space $H^{1}$, Numer. Funct. Anal. Optim. 26 (2005), no. 7-8, 925-952. MR2192029

60. $\ldots$ Equivalence between lowest-order mixed finite element and multi-point finite volume methods on simplicial meshes, M2AN Math. Model. Numer. Anal. 40 (2006), no. 2, 367-391. MR 2241828 (2007e:76192)

61. tions of convection-diffusion-reaction equations, ESAIM Proc. 18 (2007), 57-69. MR2404896 (2009d:65183)

62. , A posteriori error estimates for lowest-order mixed finite element discretizations of convection-diffusion-reaction equations, SIAM J. Numer. Anal. 45 (2007), no. 4, 1570-1599. MR 2338400

63. Guaranteed and fully robust a posteriori error estimates for conforming discretizations of diffusion problems with discontinuous coefficients, HAL Preprint 00235810, version 2, submitted for publication, 2009

64. _ Residual flux-based a posteriori error estimates for finite volume and related locally conservative methods, Numer. Math. 111 (2008), no. 1, 121-158. MR.2448206

65. Martin Vohralík, Jiří Maryška, and Otto Severýn, Mixed and nonconforming finite element methods on a system of polygons, Appl. Numer. Math. 57 (2007), 176-193.

66. Mary F. Wheeler and Ivan Yotov, A posteriori error estimates for the mortar mixed finite element method, SIAM J. Numer. Anal. 43 (2005), no. 3, 1021-1042. MR2177794(2006h:65174)

67. Barbara I. Wohlmuth and Ronald H. W. Hoppe, A comparison of a posteriori error estimators for mixed finite element discretizations by Raviart-Thomas elements, Math. Comp. 68 (1999), no. 228, 1347-1378. MR.1651760 (99m:65222)

68. Anis Younès, Philippe Ackerer, and Guy Chavent, From mixed finite elements to finite volumes for elliptic PDEs in two and three dimensions, Internat. J. Numer. Methods Engrg. 59 (2004), no. 3, 365-388. MR2029282

69. Olgierd C. Zienkiewicz and Jian Z. Zhu, A simple error estimator and adaptive procedure for practical engineering analysis, Internat. J. Numer. Methods Engrg. 24 (1987), no. 2, 337-357. MR875306 (87m:73055)

UPMC Université Paris 06, UMR 7598, Laboratoire Jacques-Louis Lions, 75005, Paris, France -And- CNRS, UMR 7598, Laboratoire Jacques-Louis Lions, 75005, Paris, FRANCE

E-mail address: vohralik@ann.jussieu.fr 